\title{
Bose-Fermi competition in holographic metals
}

\author{
Yan Liu, Koenraad Schalm, Ya-Wen Sun, Jan Zaanen \\ Institute Lorentz for Theoretical Physics, Leiden University \\ P.O. Box 9506, Leiden 2300RA, The Netherlands \\ E-mail: liu, kschalm, sun, jan@lorentz.leidenuniv.nl.
}

\begin{abstract}
We study the holographic dual of a finite density system with both bosonic and fermionic degrees of freedom. There is no evidence for a universal bose-dominated ground state. Instead, depending on the relative conformal weights the preferred groundstate is either pure AdS-Reissner-Nordstrom, a holographic superconductor, an electron star, or a novel mixed state that is best characterized as a hairy electron star.
\end{abstract}




\section{Contents}

1 Introduction 1

2 Set-up 2

2.1 Fermions in the fluid approximation 3

2.2 Homogenous solutions to the Charged Fluid-Scalar-Gravity system in AdS 5

$\begin{array}{lll}3 & \text { Zero temperature solutions } & 7\end{array}$

$\begin{array}{lll}3.1 & \text { IR Stability analysis } & 8\end{array}$

3.2 The new Hairy Electron Star solution $\quad 9$

$4 \quad T=0$ Phase diagram $r$

$\begin{array}{lll}4.1 & \text { Quantum phase transition boundaries } & 18\end{array}$

$\begin{array}{lll}4.2 & \text { The critical point } & 25\end{array}$

5 Conclusion and discussion $\quad 26$

\section{Introduction}

It is a rule of thumb that at any given finite density bosons always win from fermions. Many bosons can coherently occupy the groundstate whereas the Gibbs potential gain decreases with each additional fermion due to the Pauli principle. At the same time it is not difficult to construct a system where this notion does not hold. A relativistic system with massive bosons but massless fermions, will first occupy the fermionic modes until the chemical potential reaches the mass of boson, or if there is an incredibly large number of distinguishable degenerate fermions, Pauli blocking is not relevant.

Strongly coupled system with no clear particle spectrum, e.g. conformal field theories, are another system where the validity of this rule is not obvious. Using the insight offered by the AdS/CFT correspondence, we study here combined Bose-Fermi systems at finite density. ${ }^{1}$ For each separately AdS/CFT has already given us some remarkable if not revolutionary insights: one can describe the condensation of strongly coupled bosonic systems at finite density with order parameter dimensions that are far beyond perturbation theory [5-8]. Fermionic AdS/CFT systems naturally describe non-Fermi-liquid states [9-11]. The origin of this exotic physics can be traced to the interplay between the charged sector exposed by the chemical potential and a large neutral critical sector that survives in the deep IR [12]. Standard generic condensed matter wisdoms are recovered when this sector is lifted. This removes the strongly coupled particle-less physics from the deep IR.

\footnotetext{
${ }^{1}$ Holographic bosonic competing systems have been studied in e.g. [1-4].
} 
We shall stay within the confines of the standard AdS/CFT set-up and study this Bose-Fermi competition in the strongly coupled regime. For the fermions we shall take a conventional fluid approximation in the bulk (Sec. 2). This is known to correspond to a large number of distinct Fermi surfaces in the dual theory [13-16]. It already indicates that the simple Bose-Fermi competition rule might not hold. Indeed we find that, depending on the charges and conformal dimensions of the bosonic and fermionic operators, a mixed regime exists (Sec. 3). The gravitational dual to this regime is an electron star with charged scalar hair and we call this a hairy electron star solution. In the section 4 we explore the phase diagram of this system at zero temperature as a function of the scaling dimensions of the bose and fermi fields respectively. We find that each of the four phases dominates a distinct region in the phase diagram. Our conclusions and discussion of the result are in section 5 .

Note added: As we were finalizing our paper, we were informed that F. Nitti, G. Policastro and T. Vanel have obtained similar results [17].

\section{Set-up}

The gravity Lagrangian encoding the strongly coupled field theory we consider is $3+1$ dimensional AdS-Einstein-Maxwell theory with a charged massive scalar and a charged massive fermion. We only consider renormalizable interactions in the bulk, corresponding to the most relevant operators in the large $N$ expansion of the field theory. For generic charges, when $q_{b} \neq 2 q_{f}$ and $q_{b} \neq 0$, there is no such renormalizable Yukawa coupling and there is no direct interaction between the bosons and fermions. In the context that we are interested in, the most general (parity conserving) gravity Lagrangian is therefore

$$
\mathcal{L}=\frac{1}{2 \kappa^{2}}\left(R+\frac{6}{L^{2}}\right)-\frac{1}{4 e^{2}} F_{\mu \nu} F^{\mu \nu}-\left|\left(\partial_{\mu}-i q_{b} A_{\mu}\right) \phi\right|^{2}-V(|\phi|)-i \bar{\Psi}\left(\Gamma^{\mu} \mathcal{D}_{\mu}-m_{f}\right) \Psi,
$$

where

$$
\begin{gathered}
V(\phi)=\frac{u}{2 L^{2}}\left(|\phi|^{2}+\frac{m_{b}^{2} L^{2}}{u}\right)^{2}-\frac{m_{b}^{4} L^{2}}{2 u}, \\
\bar{\Psi}=\Psi^{\dagger} \Gamma^{\underline{t}}, \quad \mathcal{D}_{\mu}=\partial_{\mu}+\frac{1}{4} \omega_{a b \mu} \Gamma^{a b}-i q_{f} A_{\mu} .
\end{gathered}
$$

Here $m_{b}, q_{b}$, and $m_{f}, q_{f}$, denote the mass and charge of the bosons and the fermions respectively, and $\kappa, e, u$ are the Newton constant, the Maxwell coupling constant and the $\phi^{4}$ coupling constant. Rescaling $A_{\mu} \rightarrow e A_{\mu}$ shows that the action only depends on the combinations $e q_{b}$ and $e q_{f}$ and we will use this to fix $q_{f}=1$. Preliminary results on the special case $q_{b}=0$ are given in [18] and we treat the case $q_{b}=2 q_{f}$ in which case a Yukawa coupling is allowed in a companion article [19, 20].

Our main aim is to examine the zero temperature groundstates for different values of these parameters. This implies that we need to solve the full equations of motion of this 
system including the backreactions of the gauge field and the matter fields on the geometry. These equations of motion are

$$
\begin{aligned}
R_{\mu \nu}-\frac{1}{2} g_{\mu \nu} R-\frac{3}{L^{2}} g_{\mu \nu} & =\kappa^{2}\left[T_{\mu \nu}^{\text {gauge }}+T_{\mu \nu}^{\text {fermion }}+T_{\mu \nu}^{\text {boson }}\right], \\
\nabla_{\nu} F^{\mu \nu} & =e^{2}\left[J_{\text {boson }}^{\mu}+J_{\text {fermion }}^{\mu}\right], \\
\left(\nabla^{\mu}-i q_{b} A^{\mu}\right)\left(\nabla_{\mu}-i q_{b} A_{\mu}\right) \phi-\frac{\phi}{2|\phi|} V^{\prime}(|\phi|) & =0 \\
i\left(\Gamma^{\mu} \mathcal{D}_{\mu}-m_{f}\right) \Psi & =0
\end{aligned}
$$

where

$$
\begin{aligned}
T_{\mu \nu}^{\text {gauge }}= & \frac{1}{e^{2}}\left(F_{\mu \rho} F_{\nu}{ }^{\rho}-\frac{1}{4} F^{2} g_{\mu \nu}\right) \\
T_{\mu \nu}^{\text {fermion }}= & \frac{1}{2}\left\langle i \bar{\Psi} \Gamma_{(\mu} \mathcal{D}_{\nu)} \Psi-i \bar{\Psi} \overleftarrow{\mathcal{D}}_{(\mu} \Gamma_{\nu)} \Psi\right\rangle, \\
T_{\mu \nu}^{\text {boson }}= & \left(\partial_{\mu}+i q_{b} A_{\mu}\right) \phi^{*}\left(\partial_{\nu}-i q_{b} A_{\nu}\right) \phi+\left(\partial_{\mu}-i q_{b} A_{\mu}\right) \phi\left(\partial_{\nu}+i q_{b} A_{\nu}\right) \phi^{*} \\
& \quad-g_{\mu \nu}\left[\left|\left(\partial_{\alpha}-i q_{b} A_{\alpha}\right) \phi\right|^{2}+V(|\phi|)\right], \\
J_{\text {fermion }}^{\mu}= & -q_{f}\left\langle\bar{\Psi} \Gamma^{\mu} \Psi\right\rangle, \\
J_{\text {boson }}^{\mu}= & -i q_{b}\left[\phi^{*}\left(\partial^{\mu}-i q_{b} A^{\mu}\right) \phi-\phi\left(\partial^{\mu}+i q_{b} A^{\mu}\right) \phi^{*}\right]
\end{aligned}
$$

with $A_{(\mu} B_{\nu)}=\frac{1}{2}\left(A_{\mu} B_{\nu}+A_{\nu} B_{\mu}\right)$ and $\bar{\Psi} \overleftarrow{\mathcal{D}}_{\mu}=\partial_{\mu} \bar{\Psi}+\frac{1}{4} \omega_{a b \mu} \bar{\Psi} \Gamma^{a b}+i q_{f} A_{\mu} \bar{\Psi}$. The conventions for $\Gamma$-matrices that we use in this paper are

$$
\Gamma^{\underline{t}}=\left(\begin{array}{cc}
i \sigma^{1} & 0 \\
0 & i \sigma^{1}
\end{array}\right), \quad \Gamma^{\underline{\underline{x}}}=\left(\begin{array}{cc}
-\sigma^{3} & 0 \\
0 & -\sigma^{3}
\end{array}\right), \quad \Gamma^{\underline{x}}=\left(\begin{array}{cc}
-\sigma^{2} & 0 \\
0 & \sigma^{2}
\end{array}\right), \quad \Gamma^{\underline{y}}=\left(\begin{array}{cc}
0 & \sigma^{2} \\
\sigma^{2} & 0
\end{array}\right) .
$$

\subsection{Fermions in the fluid approximation}

The inherent quantum nature of the fermions means the sources for the backreaction on the geometry and the gauge field are really expectation values. There are several ways to approximate these expectation values and incorporate the backreactions of the fermions in the bulk, which correspond to fermions in different limits: the semi-classical electron star construction with fermions in the fluid approximation limit [13, 21], the quantum electron star with fermions treated quantum mechanically [22-25]. ${ }^{2}$ We shall stay in the semiclassical approximation in the following and treat the fermions in the Thomas-Fermi fluid approximation limit. This is the well-known Tolman-Oppenheimer-Volkov construction of self-gravitating stars. For completeness we briefly review it here. Further details are in $[13,16]$

The essence of the fluid approximation is adiabaticity of the radial dependence of the chemical potential, together with a Thomas-Fermi approximation where we take the number of fermions to infinity while sending the level spacing to zero. We thus assume that the local chemical potential varies so slowly $\partial_{r} \mu_{\text {local }}(r) \ll \mu_{\text {local }}(r)^{2}$ that we can consider the contribution of fermions as if in a flat homogeneous spacetime.

\footnotetext{
${ }^{2}$ At finite temperature one can also resort to a single wavefunction Dirac hair limit [26] where the total charge is carried by a single radial fermion wavefunction.
} 
And in the Thomas-Fermi limit the stress-tensor and charge density of the fermions take the ideal fluid form

$$
T_{\mu \nu}^{\text {fermion }}=(\rho+p) u_{\mu} u_{\nu}+p g_{\mu \nu}, \quad J_{\mu}=-q_{f}\left\langle\bar{\Psi} \Gamma_{\mu} \Psi\right\rangle=q_{f} n u_{\mu},
$$

with $u_{t}=e_{t \underline{t}}=-\sqrt{-g_{t t}}$ the local Fermi-fluid velocity. The energy density $\rho$, the pressure $p$ and the number density $n$ follow directly from an integral over the now infinitesimally spaced density of states

$$
\begin{gathered}
D(\omega)=\frac{1}{\pi^{2}} \omega \sqrt{\omega^{2}-m_{f}^{2}} . \\
\rho=\int_{m_{f}}^{\mu} \omega D(\omega) d \omega, \quad n=\int_{m_{f}}^{\mu} D(\omega) d \omega, \quad p=\mu n-\rho,
\end{gathered}
$$

In the adiabatic approximation the chemical potential $\mu$ is promoted to local variable, whose evolution is self-consistently determined from the equations of motion. A famed characteristic of such self-gravitating semi-classical stars is that all these fluid parameters will vanish when $\mu \leq m_{f}$. The radial value that corresponds to this value of $\mu(r)$ is the edge of the star where the full fluid energy, charge density and pressure vanishes.

As we shall search for solutions to the equations of motion we are implicitly in the semi-classical gravity approximation. For this to be valid including the backreactions of the matter fields, we need $\kappa / L \ll 1$ and $\kappa^{2} T_{\mu \nu} \sim \mathcal{O}(1)$. This implies

- As $\kappa^{2} T_{\mu \nu}^{\text {fermion }} \sim \mathcal{O}(1)$, we have $\rho, p \sim(\kappa L)^{-2}$.

- From (2.9), we have $\mu \sim(\kappa L)^{-1 / 2}$ and $m_{f} \sim(\kappa L)^{-1 / 2}$.

- From $\kappa^{2} T_{\mu \nu}^{\text {gauge }} \sim \mathcal{O}(1)$, we have $A_{t} \sim e L \kappa^{-1}$.

- From $\kappa^{2} T_{\mu \nu}^{\text {boson }} \sim \mathcal{O}(1)$, we have $\phi \sim 1 / \kappa, u \sim \kappa^{2}, m_{b} \sim 1 / L$ and $q_{b} \sim \kappa e^{-1} L^{-1}$.

As $\mu$ is also given by $\mu \sim A_{\underline{t}}$, we must have that

$$
e^{2} \sim \kappa / L \ll 1
$$

It is convenient to rescale all fields and parameters according to their orders in $\kappa, e$ and $L$ as follows

$$
\begin{gathered}
p=\frac{1}{\kappa^{2} L^{2}} \hat{p}, \quad \rho=\frac{1}{\kappa^{2} L^{2}} \hat{\rho}, \quad n=\frac{1}{e \kappa L^{2}} \hat{n}, \quad A_{\mu}=\frac{e L}{\kappa} \hat{A}_{\mu}, \\
u=\kappa^{2} \hat{u}, \quad m_{b}=\frac{1}{L} \hat{m}_{b}, \quad m_{f}=\frac{e}{\kappa} \hat{m}_{f}, \quad \phi=\frac{1}{\kappa} \hat{\phi}, \quad \mu=\frac{e}{\kappa} \hat{\mu}, \quad q_{b}=\frac{\kappa}{e L} \hat{q}_{b} .
\end{gathered}
$$

Thus $V(|\phi|)=\frac{1}{\kappa^{2} L^{2}} \hat{V}(|\hat{\phi}|)$ turns into

$$
\hat{V}(|\hat{\phi}|)=\frac{\hat{u}}{2}\left(|\hat{\phi}|^{2}+\frac{\hat{m}_{b}^{2}}{\hat{u}}\right)^{2}-\frac{\hat{m}_{b}^{4}}{2 \hat{u}}
$$


and the fluid parameters become

$$
\hat{\rho}=\beta \int_{\hat{m}_{f}}^{\hat{\mu}} \epsilon^{2} \sqrt{\epsilon^{2}-\hat{m}_{f}^{2}} d \epsilon, \quad \hat{n}=\beta \int_{\hat{m}_{f}}^{\hat{\mu}} \epsilon \sqrt{\epsilon^{2}-\hat{m}_{f}^{2}} d \epsilon, \quad \hat{p}=\hat{\mu} \hat{n}-\hat{\rho},
$$

where

$$
\beta=\frac{e^{4} L^{2}}{\pi^{2} \kappa^{2}}
$$

is an $\mathcal{O}(1)$ number.

This rescaling procedure in fact rescales $\kappa$ and $L$ out of the equations of motion, and only leaves $\beta$ (or alternatively $e$ ), $\hat{q}_{b}, \hat{m}_{f}, \hat{m}_{b}^{2}$ and $\hat{u}$ as parameters. Notably no terms in the equations of motions are irrelevant in this semiclassical limit.

\subsection{Homogenous solutions to the Charged Fluid-Scalar-Gravity system in AdS}

To solve the equations of motion, we make the following homogeneous ansatz of the metric, the gauge field and the matter fields

$$
d s^{2}=L^{2}\left(-f(r) d t^{2}+g(r) d r^{2}+r^{2}\left(d x^{2}+d y^{2}\right)\right), \quad \hat{A}_{t}=h(r), \quad \hat{\phi}=\hat{\phi}(r) .
$$

The equations of motion become

$$
\begin{aligned}
& \frac{h^{\prime 2}}{2 f}+\frac{f^{\prime}}{r f}-g(3+\hat{p}-\hat{V})+\frac{1}{r^{2}}-\hat{\phi}^{\prime 2}-\frac{\hat{q}_{b}^{2} g h^{2} \hat{\phi}^{2}}{f}=0 \\
& \frac{1}{r}\left(\frac{f^{\prime}}{f}+\frac{g^{\prime}}{g}\right)-g \hat{\mu} \hat{n}-2 \hat{\phi}^{\prime 2}-\frac{2 \hat{q}_{b}^{2} g h^{2} \hat{\phi}^{2}}{f}=0 \\
& h^{\prime \prime}-\frac{h^{\prime}}{2}\left(\frac{f^{\prime}}{f}+\frac{g^{\prime}}{g}-\frac{4}{r}\right)-\sqrt{f} g \hat{n}-2 \hat{q}_{b}^{2} g h \hat{\phi}^{2}=0, \\
& \hat{\phi}^{\prime \prime}+\frac{\hat{\phi}^{\prime}}{2}\left(\frac{f^{\prime}}{f}-\frac{g^{\prime}}{g}+\frac{4}{r}\right)-\frac{1}{2} g \frac{\partial \hat{V}}{\partial \hat{\phi}}+\frac{\hat{q}_{b}^{2} g h^{2} \hat{\phi}}{f}=0 .
\end{aligned}
$$

In addition there is a constraint from the energy momentum conservation

$$
-2 \sqrt{f} \hat{n} h^{\prime}+\hat{\mu} \hat{n} f^{\prime}+2 f \hat{p}^{\prime}=0 .
$$

Substituting the fluid expressions (2.14) into the above equation, one obtains the relation between the local chemical potential and the electrostatic potential

$$
\hat{\mu}=\frac{h+C}{\sqrt{f}} .
$$

We will set the constant $C=0$ to avoid possible singularities.

We shall search for solutions which are asymptotically $\mathrm{AdS}_{4}$. When $r \rightarrow \infty$, as $\hat{\mu} \rightarrow 0$ all the fluid parameters $\hat{\rho}, \hat{p}, \hat{n}$ vanish. The asymptotic behavior can therefore be analyzed in the framework of Einstein-Maxwell-Scalar gravity. This is well known [6-8]. For a 
"light" scalar field with $-9 / 4<\hat{m}_{b}^{2}<-5 / 4$ the behavior of the fields near the conformal $\mathrm{AdS}_{4}$ boundary is

$$
\begin{aligned}
f & =c^{2}\left(r^{2}-\frac{E-\left(\frac{4}{3} \hat{m}_{b}^{2}+2 \Delta_{1}\right) \Phi_{1} \Phi_{2}}{r}\right)+\ldots \\
g & =\frac{1}{r^{2}}\left(1-\frac{\Delta_{1}}{r^{2 \Delta_{1}}} \Phi_{1}^{2}+\frac{E-2 \Delta_{1} \Phi_{1} \Phi_{2}}{r^{3}}\right)+\ldots \\
h & =c\left(\mu-\frac{Q}{r}\right)+\ldots \\
\hat{\phi} & =\frac{\Phi_{1}}{r^{\Delta_{1}}}+\frac{\Phi_{2}}{r^{\Delta_{2}}}+\ldots
\end{aligned}
$$

Here $\Delta_{1}, \Delta_{2}$ are the two roots of the relation $\Delta(\Delta-3)=\hat{m}_{b}^{2}$ and $\Delta_{1} \leq \Delta_{2}$. The scalar field has to be normalizable in this background for self-consistency. The standard boundary condition that ensures this is $\Phi_{1}=0$ which can be extended to the full semi-infinite range $\Delta_{2}>3 / 2$. An alternative boundary condition is $\Phi_{2}=0$ and is only an option within the range $1 / 2<\Delta_{1}<3 / 2$ where $1 / 2$ is the unitarity bound.

The thermodynamic properties of any particular solution are encoded in the value of its on-shell action. The bulk on shell Lagrangian can be simplified as [6]

$$
\sqrt{-g} \mathcal{L}_{\text {on-shell }}=\frac{1}{\kappa^{2}} \sqrt{-g} g^{x x} R_{x x}=-\frac{L^{2}}{\kappa^{2}}\left(\sqrt{\frac{f}{g}} r\right)^{\prime}
$$

using the equation of motion for $g_{x x}$. In addition there is also a boundary term both in the gravity and the scalar sector.

$$
\begin{aligned}
\sqrt{-\gamma} \mathcal{L}_{\mathrm{b} n d} & =\sqrt{-\gamma}\left(\frac{1}{\kappa^{2}}\left(K-\frac{2}{L}\right)-\frac{\Delta_{1}}{L} \phi^{2}\right) \\
\left.\Rightarrow \sqrt{-\gamma} \mathcal{L}_{\mathrm{b} n d}\right|_{\text {on-shell }} & =\frac{L^{2}}{\kappa^{2}} \sqrt{f} r^{2}\left(\frac{2}{r \sqrt{g}}+\frac{f^{\prime}}{2 f \sqrt{g}}-2-\Delta_{1} \hat{\phi}^{2}\right) .
\end{aligned}
$$

Thus the total on-shell action is

$$
\frac{\kappa^{2}}{L^{2} V} S_{\text {on-shell }}=c\left(E / 2-\left(2 \hat{m}_{b}^{2}+3\right) \Phi_{1} \Phi_{2}\right) .
$$

The equations of motion have a scaling symmetry

$$
r \rightarrow a r, \quad(t, x, y) \rightarrow(t, x, y) / a, \quad f \rightarrow a^{2} f, \quad, g \rightarrow g / a^{2}, \quad h \rightarrow a h
$$

with an associated Noether current

$$
J_{N}=\frac{-2 r^{2} h h^{\prime}+r^{2} f^{\prime}-2 r f}{\sqrt{f g}}
$$

This can be used to derive the following useful identity: as $\partial_{r} J_{N}=0$, we have $J_{N}(r=$ $\infty)=J_{N}(r=0)$ and this gives

$$
3 E-2 \mu Q-\left(4 \hat{m}_{b}^{2}+6 \Delta_{1}\right) \Phi_{1} \Phi_{2}=0
$$


at zero temperature. ${ }^{3}$ This relation is extremal to check the consistency of the numerical solutions we shall derive below. In addition it helps expose the underlying thermodynamics in the gravity system. With the help of this identity the free energy - minus the on-shell effective action - can be rewritten in the standard zero-temperature equilibrium relation

$$
F / V=-\frac{\kappa^{2}}{c L^{2} V} S_{\text {on-shell }}=E-\mu Q .
$$

Let us briefly discuss the total charge density on the right-hand-side of the above equation. In any multi component system, it is the sum of individual contributions. In the system we study here with both fermions and bosons, the total charge density of the dual field theory is composed out of the bosonic charge density and the fermionic charge density. We can see this explicitly. The total boundary charge density can be read from the asymptotic behavior of the Maxwell field

$$
\rho_{\text {boundary }}=\left.\sqrt{-g} F^{t r}\right|_{r=\infty}=\left.\frac{h^{\prime} r^{2}}{\sqrt{f g}}\right|_{r=\infty}=Q .
$$

Inspecting the equations of motion of this system, we see we can rewrite (2.19) as

$$
Q=Q_{b}+Q_{f}
$$

where the bulk charged densities integrated along the radial direction are directly recognized as

$$
Q_{b}=\int_{0}^{\infty} d r \sqrt{-g} \frac{2 \hat{q}_{b}^{2} h \hat{\phi}^{2}}{f}
$$

and

$$
Q_{f}=\int_{0}^{\infty} d r \sqrt{-g} \frac{\hat{n}}{\sqrt{f}}
$$

In the next section we will search for solutions of this system by finding the near horizon solutions first and then integrate to the asymptotically $\mathrm{AdS}_{4}$ boundary with normalizable scalar boundary conditions.

\section{Zero temperature solutions}

We shall aim to determine the most stable homogeneous ground states at zero temperature for different parameter regions of $\left(\hat{m}_{b}, \hat{m}_{f}, \hat{q}_{b}\right)$, holding $\beta$ (or equivalently $\left.q_{f}\right)$ and $\hat{u}$ fixed to simplify the system. We shall find that in addition to the three known types of zero temperature solutions in this system: the AdS Reissner Nordström (RN) black hole $\left(T_{\mu \nu}^{\text {boson }}=\right.$ $\left.T_{\mu \nu}^{\text {fermion }}=0\right)$, the holographic superconductor solution $\left(T_{\mu \nu}^{\text {boson }} \neq 0, T_{\mu \nu}^{\text {fermion }}=0\right)$, and the electron star solution $\left(T_{\mu \nu}^{\text {boson }}=0, T_{\mu \nu}^{\text {fermion }} \neq 0\right)$, there is in addition a new kind of hairy electron star solution for which $T_{\mu \nu}^{\text {boson }} \neq 0$ and $T_{\mu \nu}^{\text {fermion }} \neq 0$.

Let us briefly summarize the three known solutions:

\footnotetext{
${ }^{3}$ At finite temperature, as $J_{N}\left(r_{\text {hor }}\right) \neq 0$ there will be an extra term in the eqn. (2.29).
} 
- The Reissner Nordström black hole (RN) is the solution to this system when no scalar field or fermionic fluid is excited. The solution is

$$
f(r)=\frac{1}{g(r)}=r^{2}\left(1+\frac{Q^{2}}{r^{4}}-\frac{1}{r^{3}}\left(r_{0}^{3}+\frac{Q^{2}}{r_{0}}\right)\right), \quad h=\frac{\sqrt{2} Q}{r_{0}}\left(1-\frac{r_{0}}{r}\right),
$$

where $r_{0}$ is the horizon, $Q$ is the charge and at zero temperature $Q=\sqrt{3} r_{0}^{2}$. The zerotemperature near horizon geometry is $\mathrm{AdS}_{2} \times \mathrm{R}^{2}$ with the $\mathrm{AdS}_{2}$ radius $L_{2}=L / \sqrt{6}$. In grand canonical enssemble, the free energy is $F / \mu^{3} \simeq-0.136$. This solution corresponds to the disordered phase of the boundary field theory.

- The holographic superconductor (HS) solution $[7,8]$ is the solution to this system where only normalizable bosons are excited. For nonzero $\hat{u}$, the near horizon geometry of zero temperature holographic superconductor solutions is Lifshitz geometry, i.e. when $r \rightarrow 0$, we have ${ }^{4}$

$$
f=r^{2 z}, \quad g=\frac{g_{0}}{r^{2}}, \quad h=h_{0} r^{z}, \quad \hat{\phi}=\hat{\phi}_{0} .
$$

The constants $\left(g_{0}, h_{0}, z, \hat{\phi}_{0}\right)$ are determined by the parameters of the system $\left(\hat{m}_{b}, \hat{u}, \hat{q}_{b}\right)$ (See eqn. (3.11)). This solution is dual to a superconducting phase at the boundary.

- The electron star (ES) solution [13] is the solution when only fermions are excited, approximated by a fermion-fluid description. The near horizon geometry is also Lifshitz like as in (3.2) but with $\hat{\phi}_{0}=0$. (See eqn. (3.18)). This is dual to a Fermi liquid with multiple Fermi surfaces at the boundary [14-16].

\subsection{IR Stability analysis}

If the rule of thumb that bosons always win is correct, then there is a quite direct way to test this with a simple stability analysis. Starting from the electron star solution we add the scalar field as a probe and check for whether it becomes unstable in the near-horizon region. We know that the holographic superconductor background is more stable than the AdS RN background when the mass of the bosons is below the BF bound in the standard quantization. The BF bound is essentially the effective mass of the scalar field in the nearhorizon region. Consider then the electron star background instead of AdS-RN. In the presence of fermions, this electron star is always more stable than the AdS-RN background at zero temperature as long as $\hat{m}_{f}<1$. As the fermions and bosons do not have a direct interaction, the relevancy for the stability analysis is that the near-horizon ES background is now charged Lifshitz. The scalar-field equation of motion in this background is

$$
\hat{\phi}^{\prime \prime}+\frac{3+z}{r} \hat{\phi}^{\prime}-\frac{g_{0}}{r^{2}}\left(\hat{m}_{b}^{2}-h_{0}^{2} \hat{q}_{b}^{2}\right) \hat{\phi}-\frac{\hat{u} g_{0}}{r^{2}} \hat{\phi}^{3}=0 .
$$

Thus we see that the BF bound instability condition for charged bosons in the electron star background is

$$
\hat{m}_{b}^{2}-h_{0}^{2} \hat{q}_{b}^{2} \leq-\frac{(2+z)^{2}}{4 g_{0}}
$$

\footnotetext{
${ }^{4}$ For $\hat{q}_{b}=0$ case [27] one should use the near horizon ansatz $\mathrm{AdS}_{2} \times \mathrm{R}^{2}$.
} 
for the standard quantization of the scalar field. Substituting the relation between the Lifshitz parameters and the fermion mass (recall that the charge is fixed to unity) one sees that for each $\hat{m}_{f}$ there is indeed a critical value of $\hat{m}_{b}^{2}$ for which the scalar condenses.

If this condensation indeed signals a transition to the pure bosonic groundstate, the holographic superconductor, then one should simultaneously see that the holographic superconductor in the presence of fermions has an instability at the same locus in the phase diagram. From the near horizon solution of holographic superconductor (3.11) we know that the local chemical potential at the horizon is

$$
\hat{\mu}_{\mathrm{loc}}=\left.\frac{h}{\sqrt{f}}\right|_{r_{\mathrm{hor}}}=h_{0}=\sqrt{1-\frac{1}{z}} .
$$

When $\hat{m}_{f}$ is less than this number the system can support a Fermi liquid. We therefore see that the instability condition for fermions in the near horizon region is

$$
\hat{m}_{f}<\sqrt{1-\frac{1}{z}} .
$$

Substituting for the Lifshitz parameters their expression in terms of the scalar properties, we can draw both instability curves in a phase diagram (Fig. 1) as a function of $\left(\hat{m}_{f}, \hat{m}_{b}^{2}\right)$. We immediately see that the two curves do not coincide, but that there ought to exist an intermediate phase where both the fermions and bosons are excited, in other words a hairy electron star. This is a new state and it corresponds to a phase which has both superconductivity and multiple Fermi surfaces. However, these fermions are not those which form the Cooper pairs responsible for this superconductivity because there is no direct BCS type interaction between them and the charges are not related. The system with BCS interactions will be studied in [19].

Now that we know this solution has to exist, we will construct it explicitly. Before we do so, however, note that the instability analysis reveals a curious aspect. Zooming in on the location where the phase boundaries intersect, we see that the fermion instability curve in the HS phase does not smoothly transition into the ES-AdS-RN phase boundary at the critical values of $\hat{m}_{b}^{2}$. This is puzzling and could mean various things, such as a missed degree of freedom. ${ }^{5}$ We will see that the explicit solution will provide the explanation.

\subsection{The new Hairy Electron Star solution}

To obtain the hairy electron star solution we follow the same procedure as used for the holographic superconductor and the electron star. We will show that the near horizon geometry in this case is also Lifshitz geometry. Then the full asymptotically AdS solutions can be obtained by turning on the irrelevant deformations near the Lifshitz fixed points (3.2).

To illustrate the commonality of the new solution with the other near-horizon Lifshitz solutions, i.e. the holographic superconductor and the electron star, let us first briefly review the details of these solutions to continue with the construction of the hairy electron

\footnotetext{
${ }^{5}$ Note that the Gibbs rule forbidding a quadruple point does not apply as we have multicomponent system.
} 

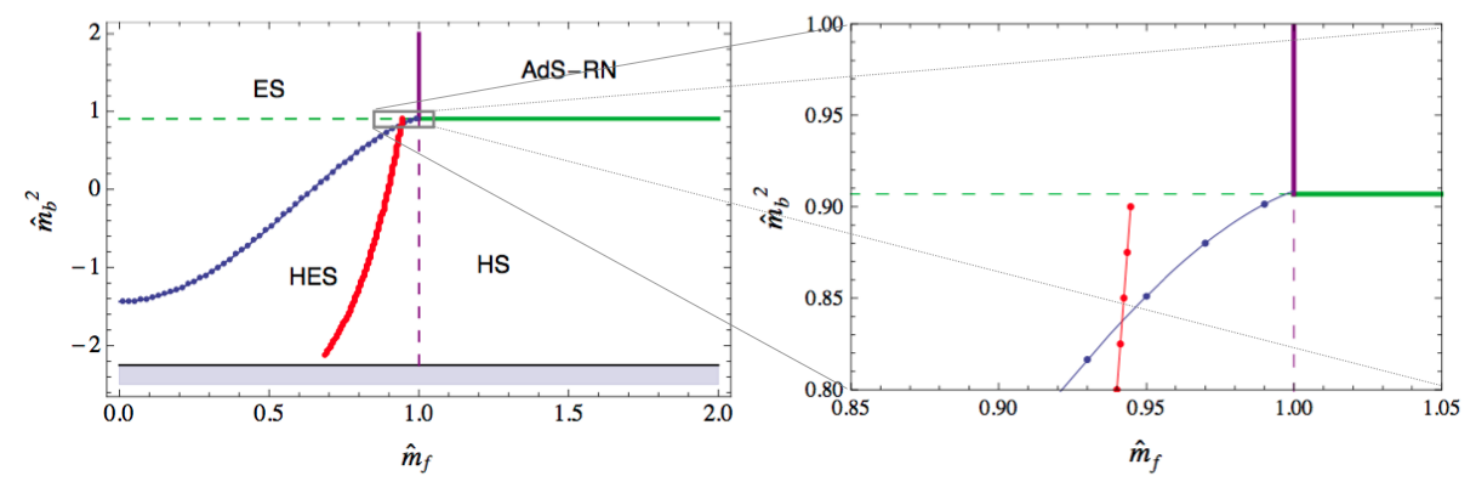

Figure 1. The instability curves for fermions and standard quantization scalars in the $\hat{m}_{b}^{2}-\hat{m}_{f}$ plane. For continuous phase transitions this should be the phase diagram. Here we fix: $\left(\hat{q}_{b}, \hat{u}, \beta\right) \simeq$ $(1.55,6,19.951)$. The green solid line gives the phase transition between AdS-RN and HS and the purple solid line is the phase transition between AdS-RN and ES. For illustration the green and purple dashed line are the extensions of the corresponding solid line. Left: blue dotted line characterizes the instability for scalar in ES. When $\hat{q}_{b}$ changes slightly, the phase diagram will also change quantitatively, but the qualitative feature stays the same except at $\hat{q}_{b}=0$. The red dotted line is the fermionic BF bound in the near horizon HS region. This gives rise to a puzzle which we shall resolve by computing the exact phase diagram. The puzzle is highlighted on the right: a magnification of the gray-box region in the left figure where the various phases meet. It is seen that the red dotted line (the BF bound) does not cross the other three boundaries at the critical point, whereas continuity between HES and ES would argue that it should.

star solution. In the subsequent section we will analyze the thermodynamics of all solutions leading to the phase diagram.

The Holographic Superconductor: In the absence of fermions the equations of motion simplify to [6-8]

$$
\begin{aligned}
\frac{h^{\prime 2}}{2 f}+\frac{f^{\prime}}{r f}-g(3-\hat{V})+\frac{1}{r^{2}}-\hat{\phi}^{2}-\frac{\hat{q}_{b}^{2} g h^{2} \hat{\phi}^{2}}{f} & =0, \\
\frac{1}{r}\left(\frac{f^{\prime}}{f}+\frac{g^{\prime}}{g}\right)-2 \hat{\phi}^{\prime 2}-\frac{2 \hat{q}_{b}^{2} g h^{2} \hat{\phi}^{2}}{f} & =0, \\
h^{\prime \prime}-\frac{h^{\prime}}{2}\left(\frac{f^{\prime}}{f}+\frac{g^{\prime}}{g}-\frac{4}{r}\right)-2 \hat{q}_{b}^{2} g h \hat{\phi}^{2} & =0, \\
\hat{\phi}^{\prime \prime}+\frac{\hat{\phi}^{\prime}}{2}\left(\frac{f^{\prime}}{f}-\frac{g^{\prime}}{g}+\frac{4}{r}\right)-\frac{1}{2} g \frac{\partial \hat{V}}{\partial \hat{\phi}}+\frac{\hat{q}_{b}^{2} g h^{2} \hat{\phi}}{f} & =0 .
\end{aligned}
$$


With the ansatz (3.2), this system has a Lifshitz scaling solution ${ }^{6}$

$$
\begin{aligned}
h_{0}^{2} & =\frac{z-1}{z}, \\
\hat{\phi}_{0}^{2} & =\frac{6 z}{\hat{m}_{b}^{2} z+\hat{q}_{b}^{2}\left(3+2 z+z^{2}\right)}, \\
g_{0} & =\frac{z}{\hat{q}_{b}^{2} \hat{\phi}_{0}^{2}}=\frac{\hat{m}_{b}^{2} z+\hat{q}_{b}^{2}\left(3+2 z+z^{2}\right)}{6 \hat{q}_{b}^{2}}, \\
\hat{u} & =-\frac{\hat{m}_{b}^{4} z^{2}+\hat{m}_{b}^{2} \hat{q}_{b}^{2} z\left(4+z+z^{2}\right)-\hat{q}_{b}^{4}\left(-3+z+z^{2}+z^{3}\right)}{6 z^{2}}
\end{aligned}
$$

There is a natural constraint $z \geq 1$ to make sure that $h_{0}$ is a real constant. For fixed values of $\hat{u}$ and $\hat{m}_{b}, z$ decreases when $\hat{q}_{b}$ increases and the condition $z \geq 1$ gives a constraint on $\hat{q}_{b}$ that $\hat{q}_{b}$ has a maximum value $\hat{q}_{b, \max }\left(\hat{u}, \hat{m}_{b}\right)$ at which $z=1$.

The holographic superconductor is the domain wall solution that interpolates between asymptotic $A d S_{4}$ and this Lifshitz solution in the interior. Integrating outwards, we need to consider the following irrelevant perturbation from the near horizon Lifshitz solution to flow to $A d S_{4}$ on the boundary [7]

$$
\begin{aligned}
& f=r^{2 z}\left(1+f_{1} r^{\alpha_{1}}+f_{2} r^{\alpha_{2}}\right)+\ldots, \\
& g=\frac{g_{0}}{r^{2}}\left(1+g_{1} r^{\alpha_{1}}+g_{2} r^{\alpha_{2}}\right)+\ldots, \\
& h=h_{0} r^{z}\left(1+h_{1} r^{\alpha_{1}}+h_{2} r^{\alpha_{2}}\right)+\ldots, \\
& \hat{\phi}=\hat{\phi}_{0}\left(1+\hat{\phi}_{1} r^{\alpha_{1}}+\hat{\phi}_{2} r^{\alpha_{2}}\right)+\ldots,
\end{aligned}
$$

where $\alpha_{1}>\alpha_{2}>0$ are the roots of the sextic equation for $\alpha$

$$
\alpha(\alpha+2+z)\left(\alpha^{4}+(4+2 z) \alpha^{3}+C_{2} \alpha^{2}+C_{1} \alpha+C_{0}\right)=0
$$

where

$$
\begin{aligned}
C_{0}= & -6 \hat{m}_{b}^{2}+6 \hat{q}_{b}^{2}-\frac{6 \hat{q}_{b}^{2}}{z}+\frac{20 \hat{m}_{b}^{2} z}{3}-\frac{4 \hat{m}_{b}^{4} z}{3 \hat{q}_{b}^{2}}-\frac{4 \hat{q}_{b}^{2} z}{3}-\frac{4 \hat{m}_{b}^{2} z^{2}}{3}+\frac{2 \hat{m}_{b}^{4} z^{2}}{\hat{q}_{b}^{2}} \\
& +\frac{4 \hat{q}_{b}^{2} z^{2}}{3}+\frac{4 \hat{m}_{b}^{2} z^{3}}{3}-\frac{2 \hat{m}_{b}^{4} z^{3}}{3 \hat{q}_{b}^{2}}-\frac{2 \hat{q}_{b}^{2} z^{3}}{3}-\frac{2 \hat{m}_{b}^{2} z^{4}}{3}+\frac{2 \hat{q}_{b}^{2} z^{4}}{3} \\
C_{1}= & -8+\frac{8 \hat{m}_{b}^{2}}{3}+\frac{\hat{q}_{b}^{2}}{3}+\frac{2 \hat{q}_{b}^{2}}{z}+8 z+2 \hat{m}_{b}^{2} z+\frac{2 \hat{m}_{b}^{4} z}{3 \hat{q}_{b}^{2}}-\hat{q}_{b}^{2} z+2 z^{2}+\hat{m}_{b}^{2} z^{2} \\
& +\frac{\hat{m}_{b}^{4} z^{2}}{3 \hat{q}_{b}^{2}}-\hat{q}_{b}^{2} z^{2}-2 z^{3}+\frac{\hat{m}_{b}^{2} z^{3}}{3}-\frac{\hat{q}_{b}^{2} z^{3}}{3} \\
C_{2}= & \frac{4 \hat{m}_{b}^{2}}{3}-\frac{\hat{q}_{b}^{2}}{3}+\frac{\hat{q}_{b}^{2}}{z}+10 z+\frac{\hat{m}_{b}^{2} z}{3}+\frac{\hat{m}_{b}^{4} z}{3 \hat{q}_{b}^{2}}-\frac{\hat{q}_{b}^{2} z}{3}-z^{2}+\frac{\hat{m}_{b}^{2} z^{2}}{3}-\frac{\hat{q}_{b}^{2} z^{2}}{3} .
\end{aligned}
$$

There are two independent perturbations because the equation of motion for the scalar field is second order and we need an additional degree of freedom at the horizon to satisfy

\footnotetext{
${ }^{6}$ There is another possible solution with $\mathrm{AdS}_{4}$ near horizon geometry: $g_{0}=1, z=1, \hat{\phi}_{0}=0$, which usually has a higher free energy and we will not consider it here.
} 
the normalizability boundary condition at the boundary. It is important to note that when both $\alpha_{1}$ and $\alpha_{2}$ are real only the relative ratio of $f_{1} / f_{2}$ is nontrivial since we can rescale $f_{1}$ or $f_{2}$ to 1 by rescaling the coordinate $r$ (the sign of $f_{1}$ or $f_{2}$ still matters). The universal solution $\alpha=-2-z$ is related to making the Lifshitz background nonextremal. From the other four solutions, we pick the irrelevant ones, i.e. $\operatorname{Re} \alpha_{1}, \operatorname{Re} \alpha_{2}>0$ in order to construct an upwards flow from Lifshitz to the conformal $\mathrm{AdS}_{4}$ boundary.

As an example, consider $\left(\hat{m}_{b}^{2}, \hat{q}_{b}, \hat{u}\right)=(-2,1.55,6)$. This gives $\left(z, g_{0}, h_{0}, \hat{\phi}_{0}\right) \simeq(2,1.556,0.707,0.73)$. Then $\left(g_{1}, h_{1}, \hat{\phi}_{1}\right) \simeq(0.336,0.619,0.129) f_{1},\left(g_{2}, h_{2}, \hat{\phi}_{2}\right) \simeq(0.191,0.577,0.044) f_{2}$ and there are five possible values of $\alpha$. Two of them are real positive numbers: $\left(\alpha_{1}, \alpha_{2}\right) \simeq(1.245,0.728)$ and we select these. Then, integrating outwards for $\left(f_{1}, f_{2}\right)=(3.845,-1)$ one finds a normalizable solution for the scalar field with $\Phi_{2}=0$ near the conformal boundary. In this particular case, we have $\left(c, \Phi_{1}, \mu, Q, E\right) \simeq(4.095,1.085,2.654,5.896,10.433)$. In the grand canonical ensemble we have $F / \mu^{3} \simeq-0.279$.. For $\left(f_{1}, f_{2}\right) \simeq(10,2.16)$ one obtains the normalizable solution for the standard quantization case $\Phi_{1}=0$. Here $F / \mu^{3} \simeq-0.148$. In both the standard and alternative quantization case the qualitative behaviors of the background fields profile are the same, see Fig 2. In addition to the fields of the solution, we have drawn one other value, the local chemical potential $\mu_{\text {loc }}=h / \sqrt{f}$ experienced by fermions. The immediately notably feature is that it rises first as we move outward before it decreases. We will discuss the importance of this when we construct the electron star solution.
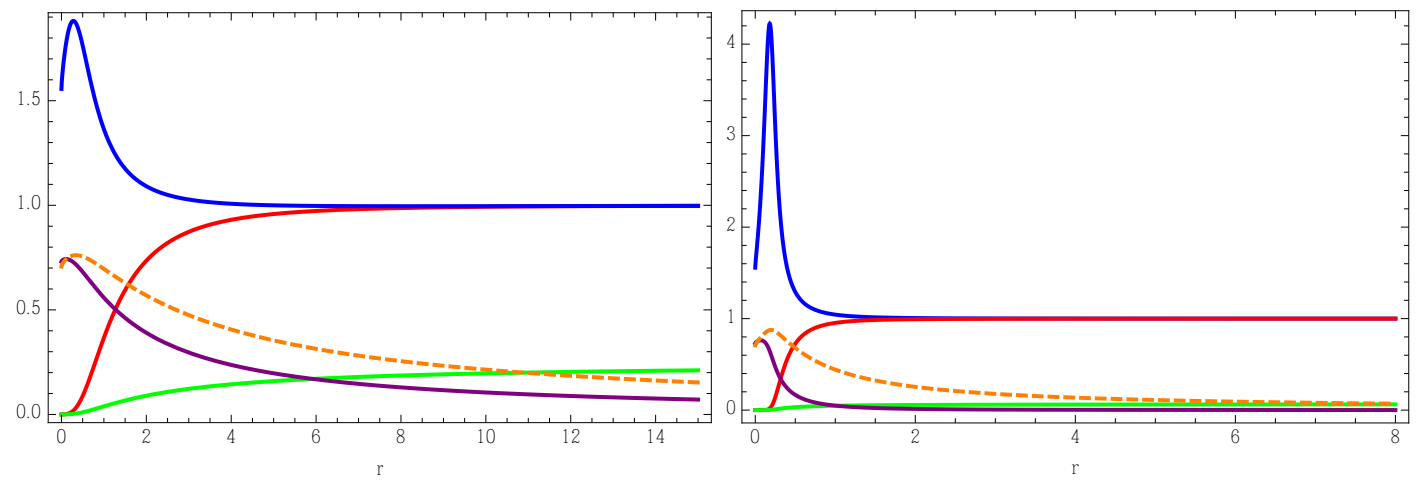

Figure 2. Background of holographic superconductor for the example mentioned in the text above: $f / c^{2} r^{2}$ (red), $g r^{2}$ (blue), $h / c \mu$ (green), $\hat{\phi}$ (purple), $\mu_{\text {loc }}$ (orange). Left: alternative quantization case; Right: standard quantization case. We can see that in both these two cases the local chemical potential has a maximum at an intermediate $r$.

The Electron Star: Without any bosonic excitations the equations of motion simplify 
to $[13,28,29]$

$$
\begin{array}{r}
\frac{h^{\prime 2}}{2 f}+\frac{f^{\prime}}{r f}-g(3+\hat{p})+\frac{1}{r^{2}}=0, \\
\frac{1}{r}\left(\frac{f^{\prime}}{f}+\frac{g^{\prime}}{g}\right)-g \hat{\mu} \hat{n}=0, \\
h^{\prime \prime}-\frac{h^{\prime}}{2}\left(\frac{f^{\prime}}{f}+\frac{g^{\prime}}{g}-\frac{4}{r}\right)-\sqrt{f} g \hat{n}=0 .
\end{array}
$$

With the ansatz (3.2) and $\hat{\phi}_{0}=0$, these equations have the Lifshitz scaling solution with

$$
h_{0}^{2}=\frac{z-1}{z}, \quad g_{0}^{2}=\frac{36 z^{4}(z-1)}{\left(\left(1-\hat{m}_{f}^{2}\right) z-1\right)^{3} \beta^{2}}
$$

and

$12\left(2+4 z+h_{0}^{2} z^{2}\right)+g_{0}\left(-72-2 h_{0}^{3} \sqrt{h_{0}^{2}-m_{f}^{2}} \beta+5 h_{0} m_{f}^{2} \sqrt{h_{0}^{2}-m_{f}^{2}} \beta\right)+3 g_{0} m_{f}^{4} \beta \log \frac{m_{f}}{h_{0}+\sqrt{h_{0}^{2}-m_{f}^{2}}}=0$.

We also have $z>1$ in this case. To flow to $A d S_{4}$ at the boundary, we again consider the irrelevant perturbations from the near horizon Lifshitz solution

$$
\begin{aligned}
f & =r^{2 z}\left(1+f_{1} r^{\alpha}\right)+\ldots, \\
g & =\frac{g_{0}}{r^{2}}\left(1+g_{1} r^{\alpha}\right)+\ldots, \\
h & =h_{0} r^{z}\left(1+h_{1} r^{\alpha}\right)+\ldots
\end{aligned}
$$

As before, $f_{1}$ can be rescaled to be 1 or -1 . A new feature is that there will be a specific edge of the star $r_{s}$ where the local chemical potential equal the mass $\frac{h\left(r_{s}\right)}{\sqrt{f\left(r_{s}\right)}}=\hat{m}_{f}$.. Beyond this value, no fermion fluid can be supported and the solution is matched on that of a standard AdS Reissner Nordstrom black hole,

$$
f=c^{2}\left(r^{2}-\frac{E}{r}+\frac{Q^{2}}{2 r^{2}}\right), \quad g=\frac{c^{2}}{f}, \quad h=c\left(\mu-\frac{Q}{r}\right) .
$$

As an example we consider $\left(\hat{m}_{f}, z, \beta\right) \simeq(0.36,2,19.951)$. In this case $\left(g_{0}, h_{0}\right) \simeq$ $(1.887,0.707)$. We have four solutions for $\alpha$. The irrelevant one is $\alpha \simeq 1.626$ and $\left(g_{1}, h_{1}\right) \simeq$ $(0.446,0.645) f_{1}$. We choose $f_{1}=-1$ and we have $\left(r_{s}, c, \mu, Q, E\right) \simeq(4.256,1.021,2.088,2.483,3.457)$. In the canonical ensemble, the free energy is $F / \mu^{3} \simeq-0.190$. The geometry background for this parameter set is plotted in Fig. 3. Note that in this zero-temperature electron star the local chemical potential is monotonically decreasing. Correspondingly there is a dense core of the star at $r=0$ which dilutes as one moves outward (Left figure in Fig 3.)

The Hairy Electron Star: Both fermions and bosons materialize and it is now required to solve the full set of equations of motion. We find it to be useful to consider the hairy star solution as an electron star solution that lives on a holographic superconducting background. As the fermions and bosons have no direct interaction this captures the 

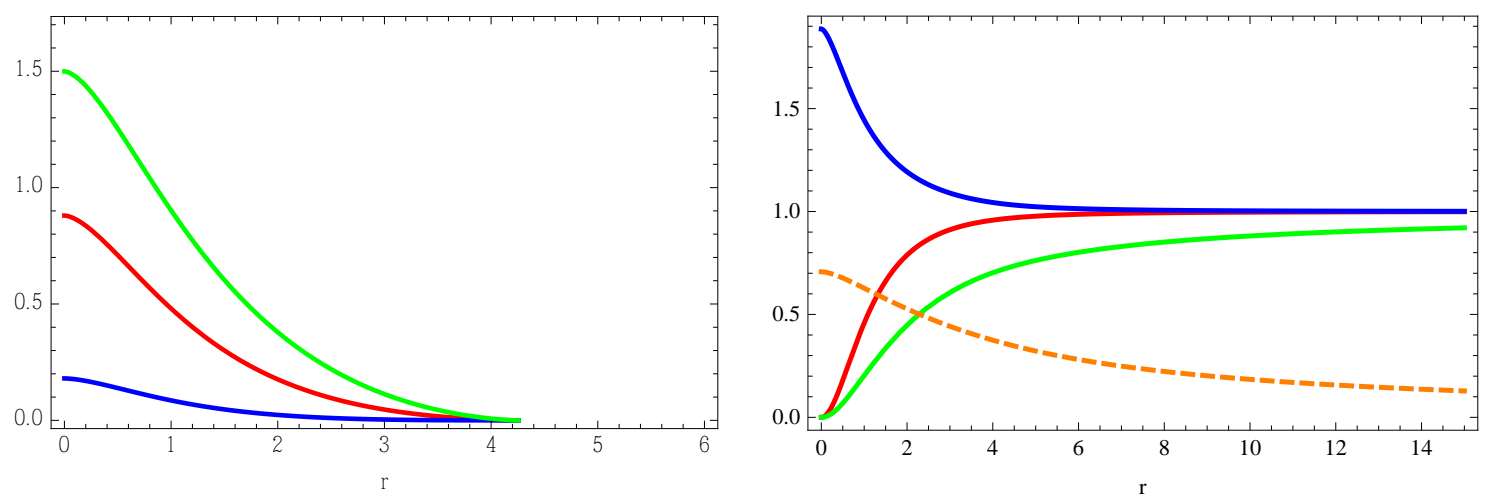

Figure 3. The background solution of an electron star for the specific example mentioned in the text above. Left: fluid profiles as functions of the radial coordinate $r: \hat{\rho}$ (red), $\hat{n}$ (green), $\hat{p}$ (blue). Right: metric background of the electron star: $f / c^{2} r^{2}$ (red), $g r^{2}$ (blue), $h / c \mu$ (green), $\mu_{\text {loc }}$ (orange).

essence of the nature of the full solution. By tuning the fermion mass downward from a high value to below the local chemical potential, the circumstances are created to form a Fermi-fluid. However, different from the pure electron star case in the zero temperature holographic superconductor background the profile of the local chemical potential is not monotonic. This type of profile is also known from finite temperature electron star solutions $[30,31]$. It implies that there are two possible kinds of hairy electron star backgrounds depending on the value of $\hat{m}_{f}$ : If $\hat{m}_{f}$ is very low, the fermi fluid can continuously exist from the interior to the outer edge. If, however, $\hat{m}_{f}$ is just below the critical value where a fermi fluid can exist, this fermi fluid has both an inner and an outer edge. This key insight is illustrated in Fig. 4.
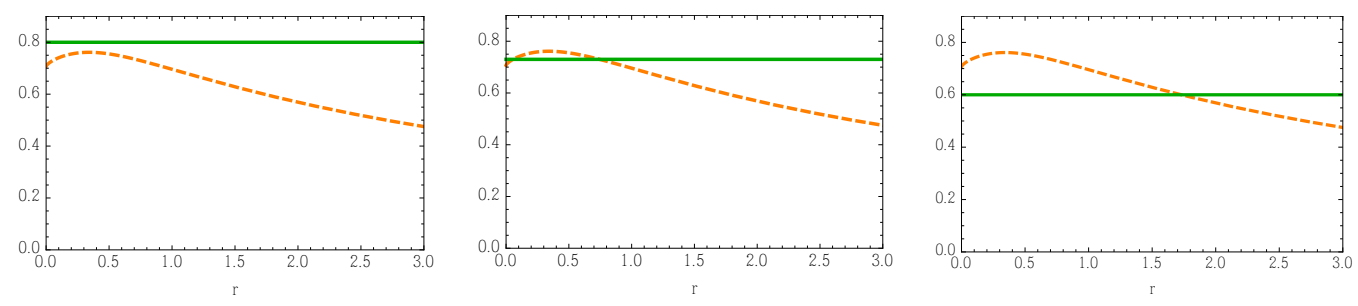

Figure 4. The orange dashed line is the local chemical potential for HS background. When we decrease $\hat{m}_{f}$ (green line) from left to right, we have HS, two-edge HES and one-edge HES solutions respectively.

CASE I: Hairy ES with one edge: For a fixed set of parameters, when we tune $\hat{m}_{f}$ to be small enough, there can always be fermonic excitations near the horizon. The near horizon parameters $\phi_{0}, g_{0}, h_{0}, z$ again are determined by the mass and charge parameters, and we do not bother to write the complicated expression out here.

As an example of hairy electron star solutions with only one edge, we consider $\left(\hat{m}_{b}^{2}, \hat{q}, \hat{u}, \hat{m}_{f}, \beta\right) \simeq$ 
$(-2,1.55,6,0.36,19.951) .{ }^{7}$ For the Lifshitz parameters we have $\left(z, g_{0}, h_{0}, \hat{\phi}_{0}\right) \simeq(1.284,1.059,0.471,0.650)$. Inside the star, the system is described by eqn. $(2.17-2.20)$.

Because there are bosons present, let us again consider irrelevant perturbations with two terms in order for the solution to flow to $A d S_{4}$ with normalizable bosons at the boundary: ${ }^{8}$

$$
\begin{aligned}
& f=r^{2 z}\left(1+f_{1} r^{\alpha_{1}}+f_{2} r^{\alpha_{2}}\right)+\ldots, \\
& g=\frac{g_{0}}{r^{2}}\left(1+g_{1} r^{\alpha_{1}}+g_{2} r^{\alpha_{2}}\right)+\ldots, \\
& h=h_{0} r^{z}\left(1+h_{1} r^{\alpha_{1}}+h_{2} r^{\alpha_{2}}\right)+\ldots, \\
& \hat{\phi}=\hat{\phi}_{0}\left(1+\hat{\phi}_{1} r^{\alpha_{1}}+\hat{\phi}_{2} r^{\alpha_{2}}\right)+\ldots
\end{aligned}
$$

The order of the equation for $\alpha$ is six and again we have the two universal solutions $\alpha_{1}=0, \alpha_{2}=-2-z$ which do not depend on the parameters we choose. In this example above, the solutions of $\alpha$ yielding irrelevant perturbations are $\alpha=0.884 \pm 0.228 i$. Usually a relevant and complex deformation $\alpha$ implies an instability [32-34]. However, it is not yet clear that an irrelevant complex deformation means an instability [7]. The one notable effect of the complex scaling dimension is that the approach to the Lifshitz fixed point is oscillatory although this is in a very small region. We will not discuss this possible instability issue or its relation on the oscillatory approach and we will assume that the absence of a relevant deformation indicates that it is a consistent and stable solution. When $\alpha_{1}$ and $\alpha_{2}$ are conjugate complex numbers, the scaling symmetry can be used to fix $f_{1}=f_{2}$ to be real.

In this specific case we have $\left(g_{1}, h_{1}, \hat{\phi}_{1}\right) \simeq(0.274+0.078 i, 0.958+0.086 i, 0.180+$ $0.176 i) f_{1}$ and $\left(g_{2}, h_{2}, \hat{\phi}_{2}\right) \simeq(0.274-0.078 i, 0.958-0.086 i, 0.180-0.176 i) f_{2}$. Note that the functions $f, g$, and $h, \phi$ are still real. For practical reason here we chose a conjugate $f_{1,2} \simeq-1 \pm 6.309 i$. The edge of the star $r_{s}$ where the fermi fluid can no longer be supported is again defined by the equality of the fermion mass with the local chemical potential

$$
\hat{m}_{f}=\frac{h\left(r_{s}\right)}{\sqrt{f\left(r_{s}\right)}},
$$

For the numerical values we find $r_{s} \simeq 0.461$.

Outside the star the system is described by Einstein-Maxwell-Scalar gravity alone (3.7 - 3.10), as in the pure holographic superconductor. At the boundary of the star $r_{s}$, we need to match the solution to the full fermion-plus-boson system to the pure boson system. This implies the boundary conditions for the outside region:

$$
\begin{aligned}
& f\left(r_{s+}\right)=f\left(r_{s-}\right), \quad g\left(r_{s+}\right)=g\left(r_{s-}\right), \quad h\left(r_{s+}\right)=h\left(r_{s-}\right), \\
& h^{\prime}\left(r_{s+}\right)=h^{\prime}\left(r_{s-}\right), \quad \hat{\phi}\left(r_{s+}\right)=\hat{\phi}\left(r_{s-}\right), \quad \hat{\phi}^{\prime}\left(r_{s+}\right)=\hat{\phi}^{\prime}\left(r_{s-}\right) .
\end{aligned}
$$

\footnotetext{
${ }^{7}$ For numerical convenience, we only consider systems with $\hat{m}_{b}^{2}=-2$ to obtain the free energies.

8 The $\hat{q}_{b}=0$ case was considered in [18]. A crucial difference is the ansatz of the perturbation of the scalar field in the near horizon region. In $\hat{q}_{b}=0$ case, from the EOM (2.20) for the scalar field, the metric and the gauge field fluctuations do not affect the scalar field at the first order of perturbation since $V^{\prime}\left(\hat{\phi}_{0}\right)=0$.
} 
We then integrate from $r_{s}$ to the boundary using the equations of motion in (3.7 - 3.10). For the values quoted above: we find after integration the $A d S_{4}$ boundary values $\left(c, \Phi_{1}, \mu, Q, E\right) \simeq$ $(1.104,0.106,0.267,0.062,0.011)$, and therefore $F=E-\mu Q \simeq-0.005$. For the grand canonical ensemble it follows that $F / \mu^{3} \simeq-0.289$. Fig. 5 shows the way that the hairy electron star solutions behave. $f, g, h, \phi$ and the fluid parameters of fermions for the parameter above.
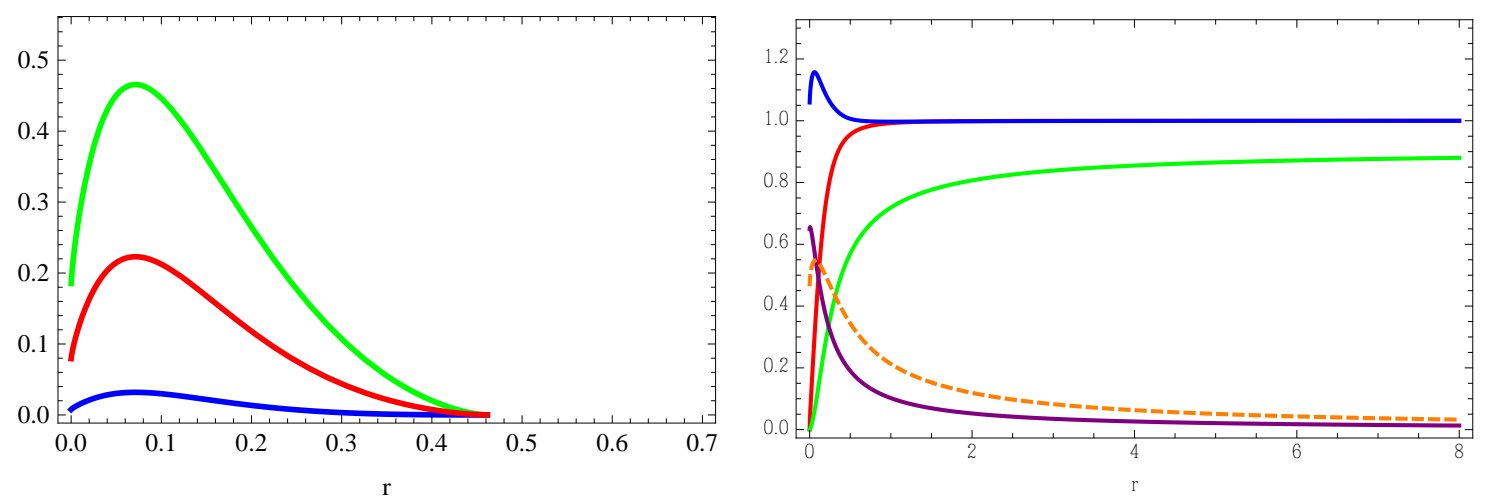

Figure 5. An example of the background of the single-edge hairy electron star solution. Left: fluid parameters as functions of the radial coordinate $r: \hat{\rho}$ (red), $\hat{n}$ (green), $\hat{p}$ (blue). It is easy to see that these functions are not monotonic along the radial coordinate as in the pure electron star case. Right: metric and scalar fields of the hairy electron star as functions of the radial coordinate $r$ : $f / c^{2} r^{2}$ (red), $g r^{2}$ (blue), $h / c \mu$ (green), $\mu_{\text {loc }}$ (orange), $\hat{\phi}$ (purple).

CASE II: hairy ES with two edges: departing from the hairy electron star with a single edge and a fermi fluid core in the interior, a different star evolves upon increasing $\hat{m}_{f}$ while the other parameters are kept fixed. The reason is that the local chemical potential in the holographic superconductor background increases first as one moves outward, before it starts to decrease. This means that when $\hat{m}_{f}$ becomes bigger than the local chemical potential in the deep interior at the horizon, it is no longer possible to support a fermifluid near the horizon. Instead an inner edge will arise where fermions start to materialize. As a typical example for this case, consider $\left(\hat{m}_{b}^{2}, \hat{q}, \hat{u}, \hat{m}_{f}, \beta\right)=(-2,1.55,6,0.725,19.951)$. Since there is no fermi fluid possible in the interior, the near horizon geometry has to be the same as the holographic superconductor. The inner edge of the star $r_{s 1}$ is defined as (3.23); for the quoted values this is at $r_{s 1} \simeq 0.064$. Then at $r_{s 1}$ we connect to the interior of the star where the system is described by full combined fermi-boson system eqn. (2.17 - 2.20) until it runs to the second edge of the star. Beyond this edge the system is again fluid-less and described by Einstein-Maxwell-scalar gravity (3.7 - 3.10). Here the outer edge is at $r=r_{s 2} \simeq 0.879$. At the asymptotical $A d S_{4}$ boundary, we obtain the values $\left(c, \Phi_{1}, \mu, Q, E\right) \simeq(3.856,1.237,3.026,7.667,15.496)$, thus $F / \mu^{3} \simeq-0.279$. In Fig. 6 we show the functions $f, g, h, \hat{\phi}$ for the fluid parameters of the specific example mentioned in the above.

Regarding the hairy electron star as a fermi fluid living on a holographic superconductor background, it is also clear that when the fermion mass $\hat{m}_{f}$ becomes larger than the 

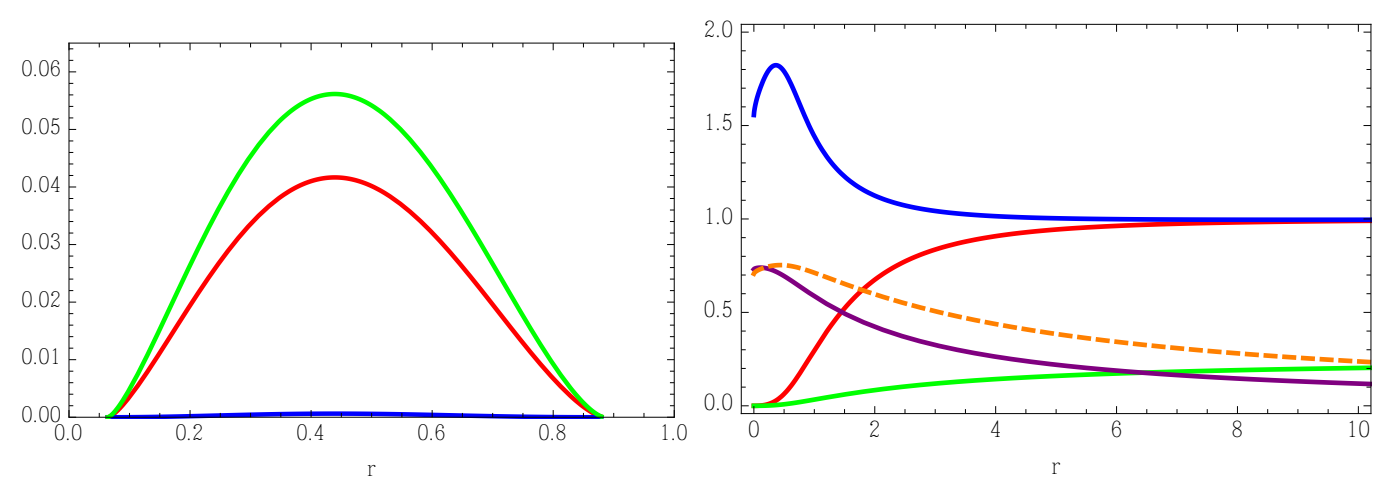

Figure 6. An example of the background of the double-edge hairy electron star solution. Left: fluid parameters as functions of the radial coordinate $r: \hat{\rho}$ (red), $\hat{n}$ (green), $\hat{p}$ (blue). We can see that there are two edges. Right: metric and scalar fields of the hairy electron star as functions of the radial coordinate $r$ : $f / c^{2} r^{2}$ (red), $g r^{2}$ (blue), $h / c \mu$ (green), $\mu_{\text {loc }}$ (orange), $\hat{\phi}$ (purple).

maximum value of the local chemical potential of the holographic superconductor background, there will be no hairy star solution anymore.

Holographic Luttinger's theorem: In principle the non-zero order parameter corresponding to non-zero vev of the scalar field signals that the field theory state dual to the hairy electron star is in a symmetry broken state. Therefore the standard Luttinger theorem need not apply. Of course since we are essentially describing a system of noninteracting bosons and fermions, there is a simple variant of the Luttinger relation. In holography one finds that the total field theory charge density equals

$$
Q_{\mathrm{FT}}=Q_{\mathrm{bulk}}+Q_{\text {horizon }}
$$

where $Q_{\text {bulk }}=\sum_{\text {occupied charged states }} Q^{(i)}$. For the pure electron star solution, where the only bulk constituents are the charged fermions the field theory Luttinger's theorem, which states that the volume of the Fermi surface is equal to the charge density of the fermions, follows from the bulk Luttinger's theorem. In Lifshitz spacetimes there is no contribution of horizon charge $[14,35]$ and thus the boundary charge density is equal to the bulk charge density. Due to the fluid approximation there will be a set of infinitely many Fermi surfaces (one for each radial mode) whose fermi momentum is the same in the bulk and in the dual field theory [14-16]. Therefore the Luttinger relation in the bulk and the boundary is the same. For the hairy electron star, the near horizon geometry is still Lifshitz with a finite $z$, so there is again no contribution of horizon charge. Also due to the fluid approximation there is a similar set of infinitely many Fermi surfaces corresponding to occupied radial modes. However, the HES solution corresponds to a condensed state with vacuum charge $Q_{b}$. But this effect can easily be accounted for. It is straightforward to see that we should now have a Luttinger relation

$$
\sum_{n} \frac{2}{(2 \pi)^{2}} V_{n}=Q-Q_{b}
$$


where $V_{n}=\pi k_{F}^{(n) 2}$ is the volume of the $n$-th Fermi surface of the dual field theory and $Q_{b}$ the boson charge density $Q_{b}=\int_{0}^{\infty} d r \sqrt{-g} 2 \hat{q}_{b}^{2} \hat{\phi}^{2} h / f$. This is familiar from fractionalized Fermi systems [36] in the broken phase. As a reservoir of charge the Bose condensate now takes over the status of the horizon charge.

\section{$4 T=0$ Phase diagram}

With the exact solutions in hand we can now construct the phase diagram at zero temperature in detail. As mentioned above, we will keep fixed $\hat{u}=6, \hat{\beta} \simeq 19.951$ and we rescaled $q_{f}$ to be 1 . The three tunable parameters we consider are $\hat{m}_{f}, \hat{m}_{b}$ and $\hat{q}_{b}$.

\subsection{Quantum phase transition boundaries}

Assuming that all the phase transitions are continuous lines of instability we determined in Fig. 1 should correspond to the exact phase boundaries. This was already known for the AdS-RN/ES phase boundary for $\hat{m}_{b}^{2} \gg 1$; in the fluid limit the nature of the transition is not known yet, but it is assumed to be continuous. ${ }^{9}$ Also the continuous AdS-RN/HS phase boundary equals the instability curve. This has been studied explicitly in [27, 34, 37] for $\hat{q}_{b}=0$ and it is BKT type. For finite $\hat{q}_{b}$ it should still be BKT and the only difference is that the near horizon geometry for the condensed phase is $\widetilde{\mathrm{AdS}}_{2}{ }^{10}$ at $\hat{q}_{b}=0$ while Lifshitz at finite $\hat{q}_{b}$. For the new phase boundaries adjacent to the new Hairy Electron Star phase, there was however a puzzle that the fermi-instability line in the holographic superconductor phase did not smoothly join the Fermi-instability line in the AdS-RN phase.

We tested the continuity of the phase transition of these new phase boundaries between HS/HES and HES/ES as well as the exact location of the phase boundary by computing the free energies of the exact solutions along the section $\hat{m}_{b}^{2}=-2$. (Other values of $\hat{m}_{b}^{2}$ are numerically hard to control). The resulting free energy of the four kinds of solutions for $\hat{q}_{b}=1.55$ as a function of $\hat{m}_{f}$ is given in Fig. 7 . What this study of the free-energy reveals is that the phase boundary between the HS and the HES solutions is not given by the deformed BF bound for fermions in the HS background. With the knowledge how the HES is constructed it is easy to see why. As we lower $\hat{m}_{f}$ from the HS reason, there is a critical value $\hat{m}_{f} \sim \mu_{\mathrm{loc}}^{\max }$ where the fluid first forms, but due to the non-monotonic behavior of $\mu_{\text {loc }}$ it does not form at the Lifshitz horizon but in the interior. The first star one encounters by tuning $\hat{m}_{f}$ down is the two-edged HES. The BF instability bound for fermions, however, is constructed from the Lifshitz scaling solution. Indeed tracing the sequence of solutions, one finds that at this value of $m_{f}$ one has a crossover from the two-edge HES to the single-edge HES.

For the free energy at value $\hat{m}_{b}^{2}=-2, \hat{q}_{b}=1.55$ the HES remains the preferred phase all the way down to $\hat{m}_{f}=0$. However, for a higher $\hat{m}_{b}^{2}$ or lower $\hat{q}_{b}$ one will encounter the transition line between the single edged HES and the ES solutions as one keeps lowering $\hat{m}_{f}$. We shall explain below that this exactly corresponds with the BF bound of bosons in

\footnotetext{
${ }^{9}$ At finite $T$ it is $3 r d$ order [30, 31].

${ }^{10}$ It is an $\mathrm{AdS}_{2}$ region with a vev for the scalar field. $\widetilde{\mathrm{AdS}}_{2}$ is used to distinguish the near horizon $\mathrm{AdS}_{2}$ geometry of RN without scalar vev.
} 


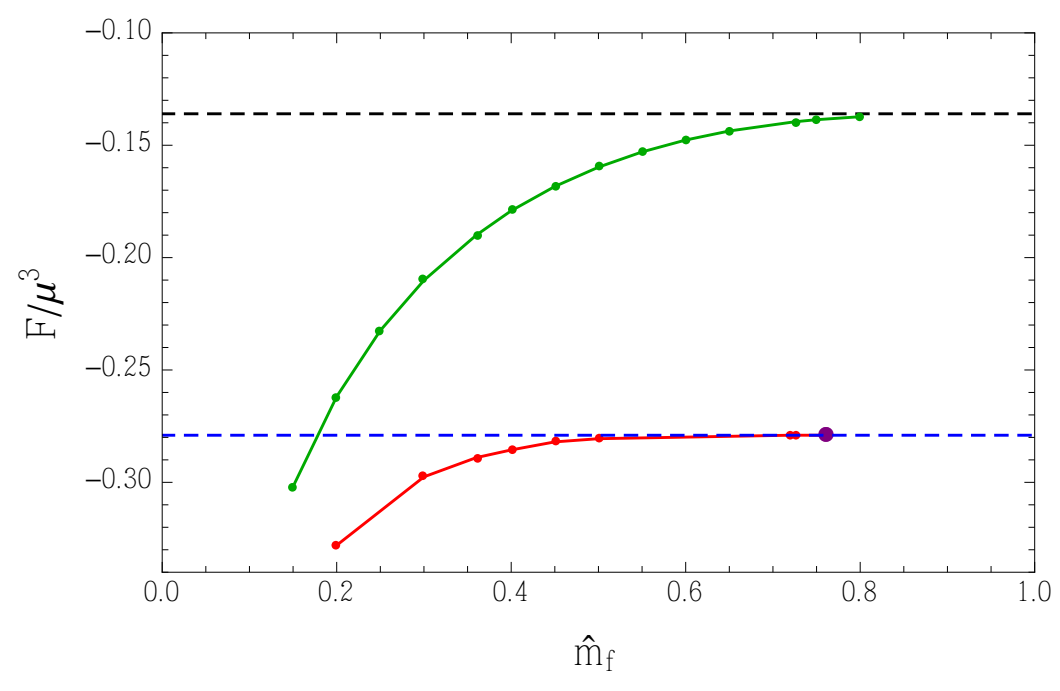

Figure 7. Free energy of the four solutions and the phase transition between HES and HS for fixed scalar mass and charge with changing fermion mass. Free energy $F / \mu^{3}$ vs. $\hat{m}_{f}$ with $\left(\hat{m}_{b}^{2}, \hat{q}_{b}, \hat{u}, \beta\right)=$ $(-2,1.55,6,19.951)$ : RN (black), ES (green), HS (blue), HES (red). The free energy of RN or HS does not depend on $\hat{m}_{f}$. The purple marked point is the bound where we can find hairy ES solution. Here we choose alternative quantization for the scalar field in HS and HES cases. For standard quantization the plot is qualitatively the same because this picture only concerns the transition between HES and HS while not the transition between ES and HES. In other words, the instability shown in this plot is only related to fermions in the HS background, so there is no qualitative dependance on the boundary condition for the scalar field. There is also no qualitative difference for other boundary conditions of the scalar field.

the ES near horizon region $\hat{m}_{b}{ }^{2}-h_{0}^{2} \hat{q}_{b}^{2}=-\frac{(z+2)^{2}}{4 g_{0}}$ (for the standard quantization of the scalar boundary condition.)

In Fig. 8 we show these results. The red dotted line in Fig. 8 is the fermion instability bound, which we now know denotes the transition between the one-edge HES and the twoedge HES. The real quantum phase transition between a HS and two-edge HES happens at the black line in the left figure. This black line can be obtained numerically by demanding $\hat{m}_{f}=\max \left[\mu_{\text {local }}(r)\right]$. It will also depend on the boundary condition of the scalar field but the dependence is only quantitative which is indicated from Fig. 2.

With this understanding of the phases for fixed $\hat{q}_{b}$, we can change this parameter as well. Nothing changes qualitatively. Specifically as one changes $\hat{q}_{b}$ one finds that

1, At $\hat{m}_{f}=0$, the value of $\hat{m}_{b}^{2}$ on the ES-HES phase boundary line is always smaller than the value of $\hat{m}_{b}^{2}$ for the HS-RN boundary as the BF bound in the ES background is always lower than the BF bound in the $\mathrm{AdS}_{2}$ background. This means that the ESHES boundary always exists and obeys the relation (3.4) no matter how $\hat{q}_{b}$ changes.

2, One important characteristic is that for $\hat{m}_{b}^{2}$ near to or equal to the unitarity bound (the bottom of the phase diagram), the single-double edge HES crossover is always on the right of the ES-HES transition. This can be seen directly from a 3D plot of 


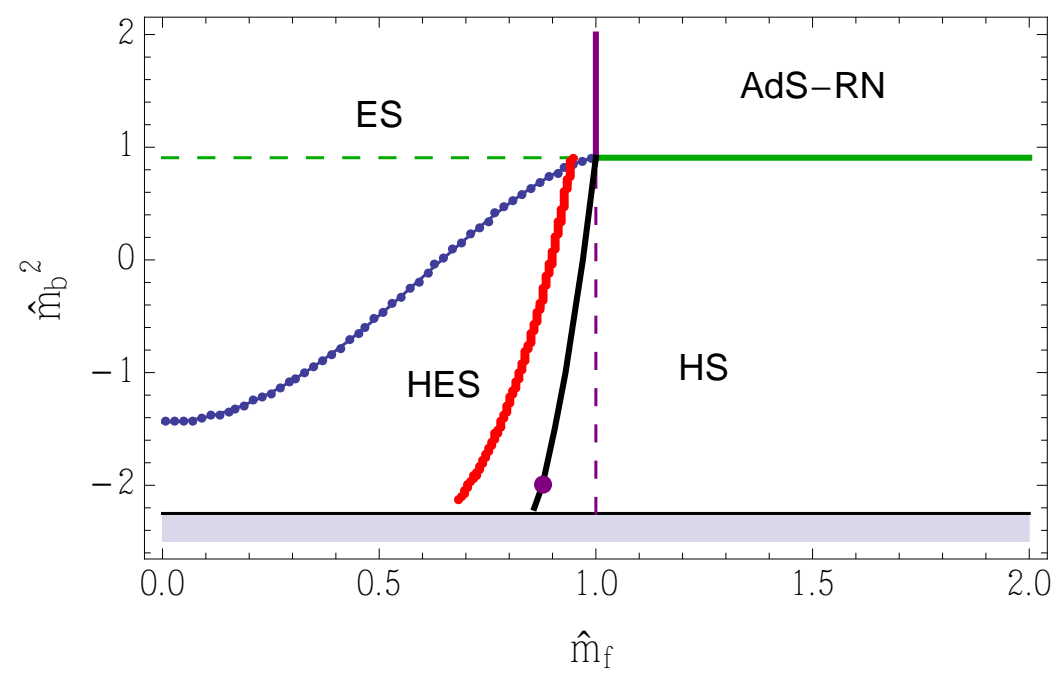

Figure 8. The improved phase diagram of Fig. 1 for $\left(\hat{q}_{b}, \hat{u}, \beta\right) \simeq(1.55,6,19.951)$. Here the red line is the incorrect instability curve from analysis of fermions in the near horizon of HS. The black line denotes the actual instabilities of fermions in HS. Due to numerical difficulties, this black line is only a rough illustration. The purple marked point is the transition point between HES and HS in the standard quantization case which is qualitatively the same to Fig. 7.

the BF stability diagram where $\hat{q}_{b}$ is taken into account. It can also be seen from the fact that the single-double edge crossover can only reach $\hat{m}_{f}=0$ for $\hat{q}_{b}>2$ while the ES-HES line always intercepts $\hat{m}_{f}=0$. From (3.4) and (3.11) we see that this intercept value of $\hat{m}_{b}^{2}$ for the ES-HES transition at $\hat{m}_{f}=0$ is always larger than that of single-double crossover. Furthermore it is immediately obvious from the construction that the double edge HES-HS transition is always to the right of singledouble HES crossover because the double-edge HES solution exists earlier than the single-edge solutions when we decrease $\hat{m}_{f}$ from the HS side. These two facts together imply that the ES-HES transition line is always above the HES-HS transition for all the possible parameter regions and the HES region always exists. There is no direct phase transition from ES to HS except at the critical point.

By construction we have quite a good understanding of the transition from the holographic superconductor to the hairy electron star. Let us finally discuss the other transition between the electron star and the hairy electron star in more detail. From the phase diagram Fig. 8 we can clearly see that the transition between ES and HES can happen both directly to the one-edge HES solution as well as to the two-edge HES solution because the ES-HES transition line intersects with the IR stability bound signaling the single-double edge crossover. These two ES-HES transitions are the same in nature but a little different in detail.

CASE I: Phase transition between ES and one-edge HES: In all cases with a 
non-trivial bosonic background the instability to forming scalar hair is a perturbative instability: the scalar perturbations on the geometry background become tachyonic modes and render the background unstable towards a new ground state. At finite temperature, even when backreactions of the scalar field to the geometry are considered, at the onset of the phase transition the scalar field is very small in the spacetime and can be treated as perturbations, so the perturbative analysis is still valid and the transition is very continuous (second order).

At zero temperature, on the other hand, one encounters the following puzzle. After taking backreaction into account, the near horizon value $\phi_{0}$ is always non-zero - it is located at the symmetry breaking minimum of the quartic potential. However, just before the transition point under any boundary condition for the scalar field, $\phi$ should vanish for a continuous phase transition, and this seems to contradict with the fact that $\phi_{0}$ just after the transition is distinctly non-zero at the horizon.

As explained in [27,34], the resolution is that there exists a special emergent IR scale for the system slightly below the BF bound and the condensate only has a finite effect below this IR scale. Near the phase transition, the dual field theory order parameter extracted from the AdS boundary value of the condensate is infinitesimal. In fact the value stays very small for a long distance along the radial direction into the interior until it reaches this special IR scale and the bulk condensate starts to become of finite size.

For the standard holographic superconductor this IR scale [27, 34] is

$$
\Lambda_{\mathrm{IR}} \sim \mu \exp \left(-\frac{c_{0}}{\sqrt{\Delta_{c}-\Delta}}\right), \quad c_{0}=\pi \sqrt{\frac{d(d-1)}{2 \Delta_{c}-d}},
$$

where $\Delta$ is the UV scaling dimension and $\Delta_{c}$ is the critical UV scaling dimension at the BF bound. This scale is exponentially small close to the BF bound, so the finite $\phi$ effect is only constrained in an exponentially small distance in the radial direction (one can think of $\Lambda_{I R}$ as the distance to the center of AdS). This makes the difference in the free energy also exponentially small, so this resolves the contradiction between a finite jump in the horizon value and a continuous phase transition between the HS and the AdS-RN black hole. In fact it shows that the phase transition is of the most continuous kind: it is a BKT phase transition. It allows one to think of the AdS geometry in the following way. In the exponentially near horizon region below this IR scale the geometry is the backreacted Lifshitz geometry, but it soon turns into an almost unaffected near horizon AdS-RN $\mathrm{AdS}_{2}$ geometry above $\Lambda_{\mathrm{I} R}$, so there exists an intermediate "semi-local quantum critical" region with the geometry of $\mathrm{AdS}_{2}$ in an intermediate scale $\Lambda_{\mathrm{IR}}$ to $\mu$.

We now show that the phase transition from HES to ES has the same feature as the transition from HS to AdS-RN. Numerically we show that near the BF bound with the standard boundary condition for the scalar field there indeed exists a intermediate region just beyond the near-horizon Lifshitz region where the scalar field drops to almost zero and the geometry in that intermediate region becomes the near horizon geometry of ES. This confirms that there exists an emergent IR scale below which the effect of the condensation becomes significant. 

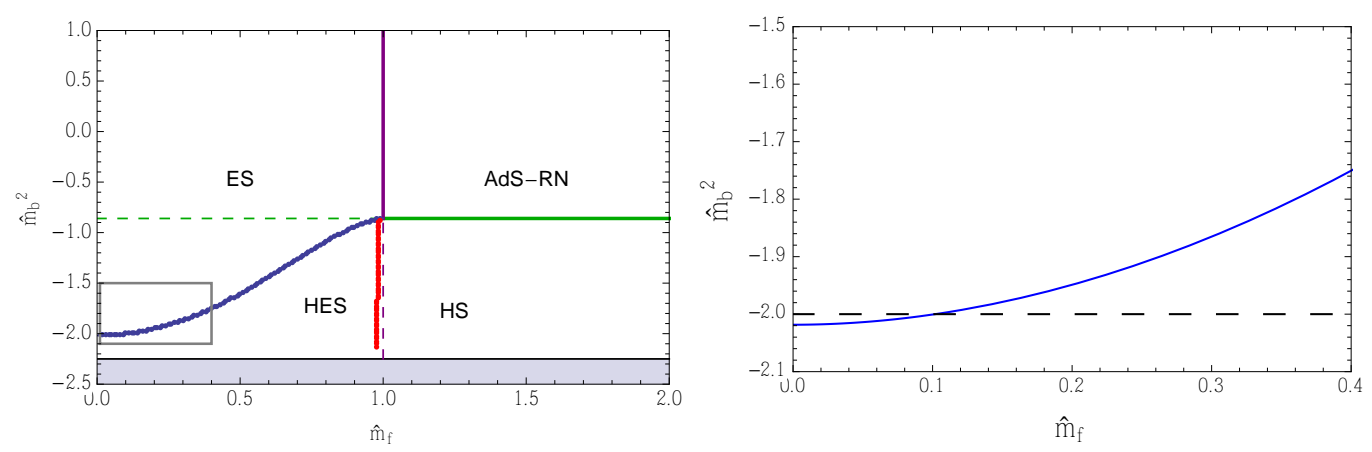

Figure 9. Left: phase diagram in the $\hat{m}_{b}^{2}-\hat{m}_{f}$ plane for $\hat{q}_{b}=0.8 ;$ Right: a clearer picture for the blue curve of the gray box region in the left figure. This is qualitatively the same as Fig. 8. The red line is the one-edge HES boundary.

To avoid numerical complexities we choose $\hat{q}_{b}=0.8$; the phase diagram for this case the phase diagram is given in Fig. 9. In Fig. 10 we show the existence of the critical region for $\left(\hat{m}_{b}^{2}, \hat{q}_{b}, \hat{m}_{f}\right)=(-2,0.8,0.12)$ and $\left(f_{1}, f_{2}\right)=(9.123,-10)$. We can see when moving outward one extremely rapidly enters an intermediate region where the blackening function $f(r)$ behaves as $r^{2 z_{\mathrm{ES}}}$ where $z_{\mathrm{ES}}$ is the Lifshitz scaling exponent of the electron star solution at this $\hat{m}_{f}$. The IR scale $r / \mu$ at which the critical regions starts to exist should be related to how close it is to the transition point and to the value of the condensate $\left\langle\mathcal{O}_{2}\right\rangle$ but here due to numerical reasons we choose the initial value of $f_{1}$ and $f_{2}$ a little away from the transition point so that the critical region starts to exist at a scale $r / \mu$ around $10^{-6}$.
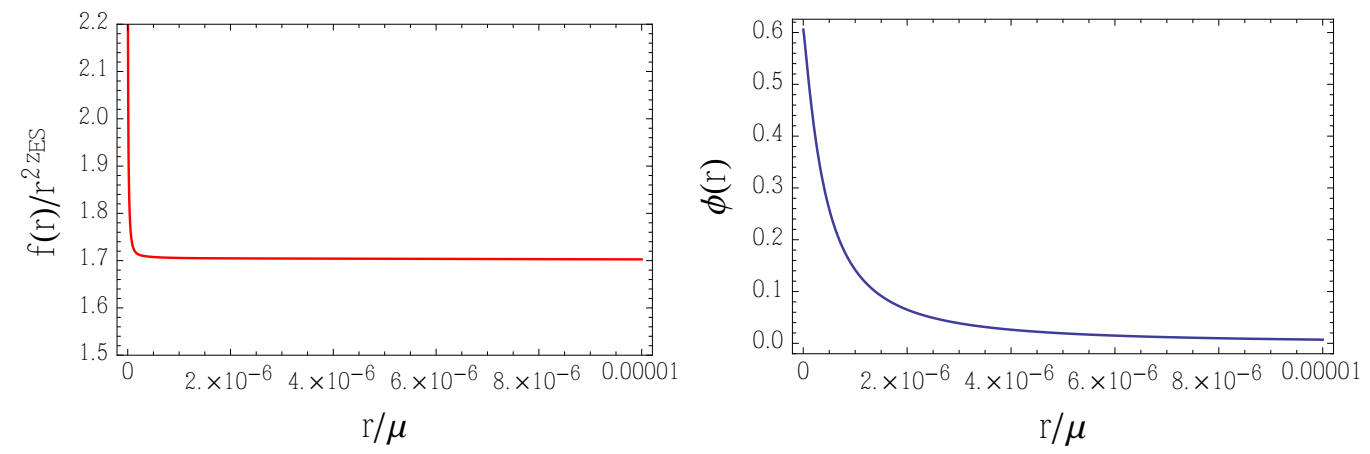

Figure 10. Evidence of the existence of an intermediate critical region for $\hat{m}_{b}^{2}=-2, \hat{q}_{b}=0.8$ and $\hat{m}_{f}=0.12$. The function of $f$ in the metric behaves as $r^{2 z_{\mathrm{E} S}}$ in an intermediate region of $r / \mu$.

In our case, the IR critical scale $[18,27]$ is

$$
\Lambda_{\mathrm{IR}} \sim \mu \exp \left(-\frac{\pi}{\sqrt{g_{0}\left(m_{c}^{2}-\hat{m}_{b}^{2}\right)}}\right),
$$

where $m_{c}^{2}=\hat{q}_{b}^{2} h_{0}^{2}-\frac{(z+2)^{2}}{4 g_{0}}$ is the mass square of the BF bound (3.4). It is easy to check 
that the difference in free energy compared to the pure electron star background should therefore scale as

$$
\delta F \sim \mu^{3} \exp \left(-\frac{(z+2) \pi}{\sqrt{g_{0}\left(m_{c}^{2}-\hat{m}_{b}^{2}\right)}}\right) .
$$

We can check this explicitly. We focus on the line of $\hat{m}_{b}^{2}=-2$ in Fig. 9 and vary $\hat{m}_{f}$ to change the BF bound $m_{c}^{2}$. In Fig.11, we plot out the curve generated by boundary values of $\Phi_{1}$ and $\Phi_{2}$ when we change the boundary values of $f_{1}$ and $f_{2}$. The different curves correspond to different values of $\hat{m}_{f}$ for fixed $\hat{m}_{b}^{2}=-2$ and $\hat{q}_{b}=0.8$, which correspond to $g_{0}\left(m_{c}^{2}-\hat{m}_{b}^{2}\right)$ ranging from -0.019 to 2.368 . For standard quantization, normalizable solutions correspond to the intersecting point of the curve with the $\Phi_{2}$ axis while for alternative quantization, normalizable solutions correspond to the intersecting point of the curve with the $\Phi_{1}$ axis. From the left figure we can see that the curves always intersect with the $\Phi_{1}$ axis far from the origin, but our interest is the intersection with the $\Phi_{2}$ axis near the origin. It is numerically difficult to get very close to the transition point, but zooming in - displayed in the right figure - we directly see that when $m_{c}^{2}-\hat{m}_{b}^{2}$ decreases the expectation value of $\Phi_{2}$ will also decrease. It is expected that $\Phi_{2}$ goes to zero when $\hat{m}_{b}^{2}$ approaches exactly $m_{c}^{2}$.

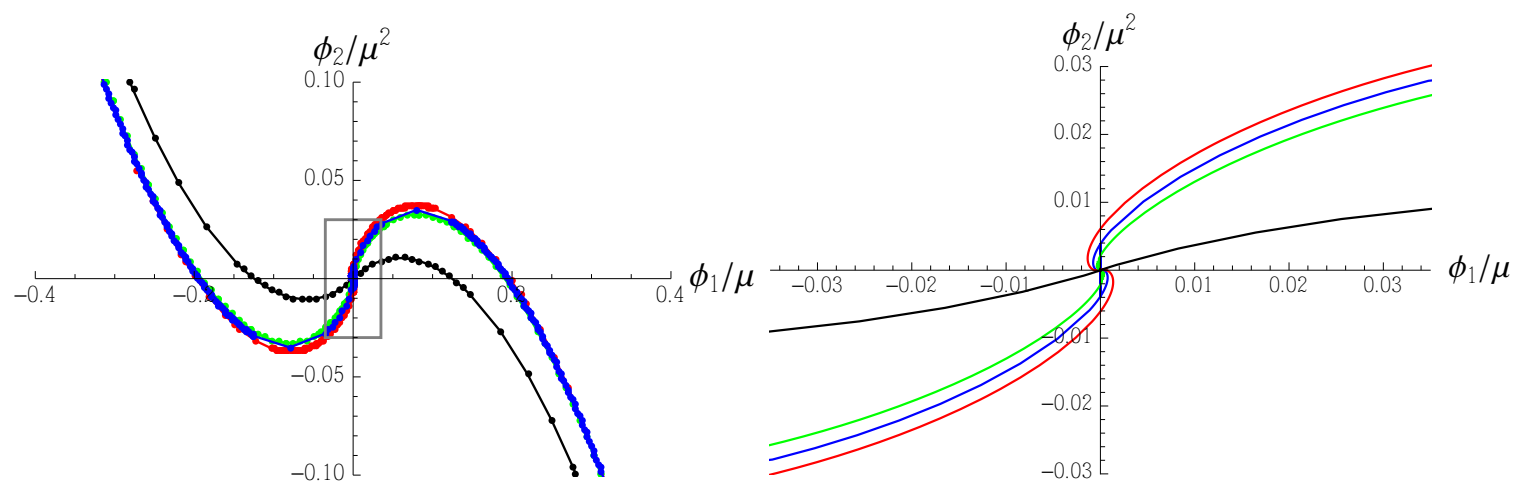

Figure 11. Plots of $\Phi_{2} / \mu^{2}$ as a function of $\Phi_{1} / \mu$ in the condensed phase (hairy ES background). Different points on each curve correspond to different values of the shooting parameters. THe right plot is the zoomed in version of the left gray boxed region. Here $\hat{m}_{f}=0.6$ (red), 0.55(blue), 0.5(green), 0.05(black), correspondingly, $g_{0}\left(m_{c}^{2}-\hat{m}_{b}^{2}\right) /(z+2)^{2}=0.074,0.061,0.046,-0.002$.

The difference of the free energy of the two phases as a function of $\hat{m}_{f}$ are shown in Fig. 12. We can see from the right figure in Fig. 12 that the free energy difference scales exactly as (4.3). Thus this phase transition is still BKT.

Note that the boundary condition for the scalar field is crucial to the behavior of the phase diagram. We can see from Fig. 11 that near the BF bound we can still find solutions with alternative boundary conditions for the scalar field with a finite expectation value $\Phi_{1}$. In fact this should be the preferred solution - in general the largest vev of either $\Phi_{1}$ or $\Phi_{2}$ is the one with lowest free energy [38]. Already for the pure holographic superconductor, but also for the hairy electron star, the free energy of the solution with alternative 

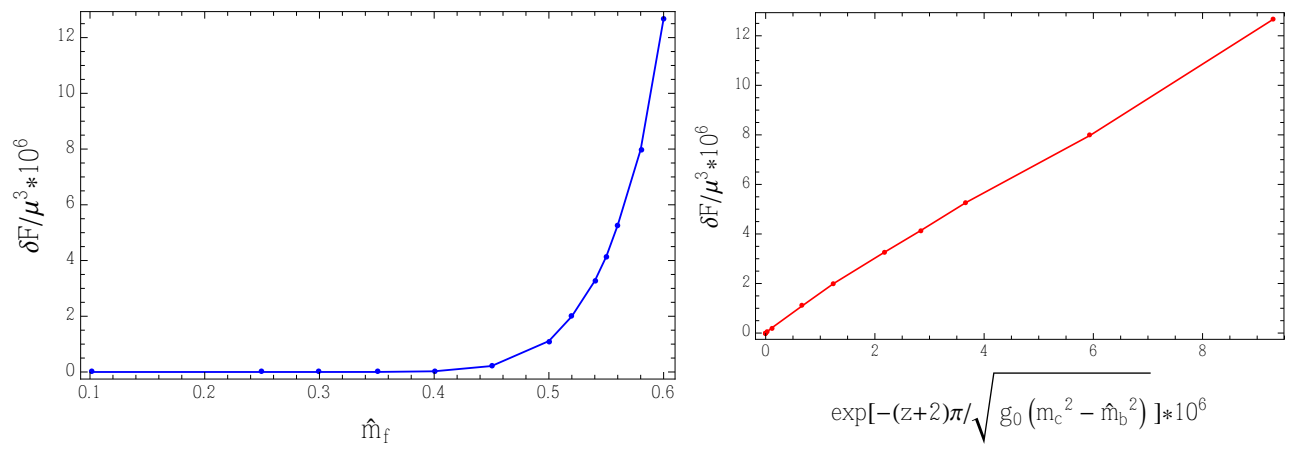

Figure 12. Difference of free energy $\delta F$ as a function of $\hat{m}_{f}$ (left) and of $\exp \left(-\frac{(z+2) \pi}{\sqrt{g_{0}\left(m_{c}^{2}-\hat{m}_{b}^{2}\right)}}\right)$ (right) for $\left(\hat{m}_{b}^{2}, \hat{q}_{b}, \hat{u}, \beta\right)=(-2,0.8,6,19.951)$ and standard boundary condition for the scalar field.

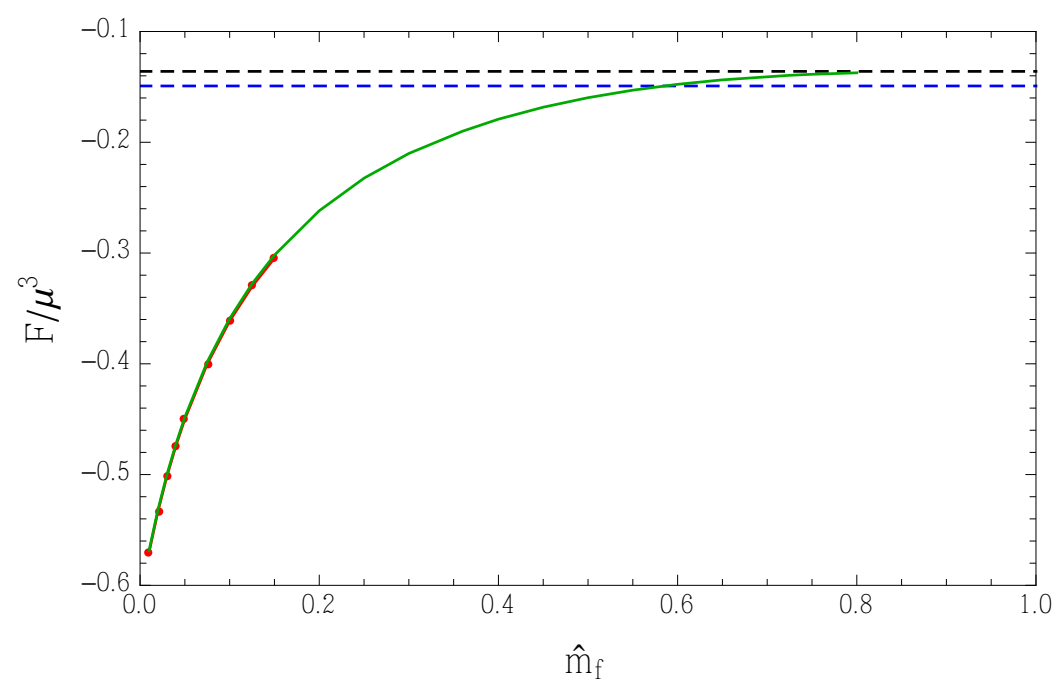

Figure 13. Free energy of HES and ES solutions for alternative boundary condition. The plot $F / \mu^{3}-\hat{m}_{f}$ with $\left(\hat{m}_{b}^{2}, \hat{q}_{b}, \hat{u}, \beta\right)=(-2,0.8,6,19.951), \mathrm{RN}$ (black), ES (green), HS (blue), HES (red): the free energy of HES always lower than ES.

boundary conditions is lower than the free energy of the solution with standard boundary condition. The same conclusion holds in the probe holographic superconductor case [38]. It is quite puzzling that for a finite density system the alternative one is more stabler as there is at the $1 / N$ level a clear double trace deformation where the zero density system should flow to standard quantization. We are not sure yet what this implies. Here we simply present the results and defer the answer to future work.

CASE II: Phase transition between ES and two-edge HES: With this knowledge we can be quick in our discussion of the phase transition between ES and the two-edge HES. It is qualitatively the same in that there are emergent IR scales. In this case moving inward for the two-edge HES the condensate starts to be significant near a similar $\Lambda_{\mathrm{IR}}$ as 


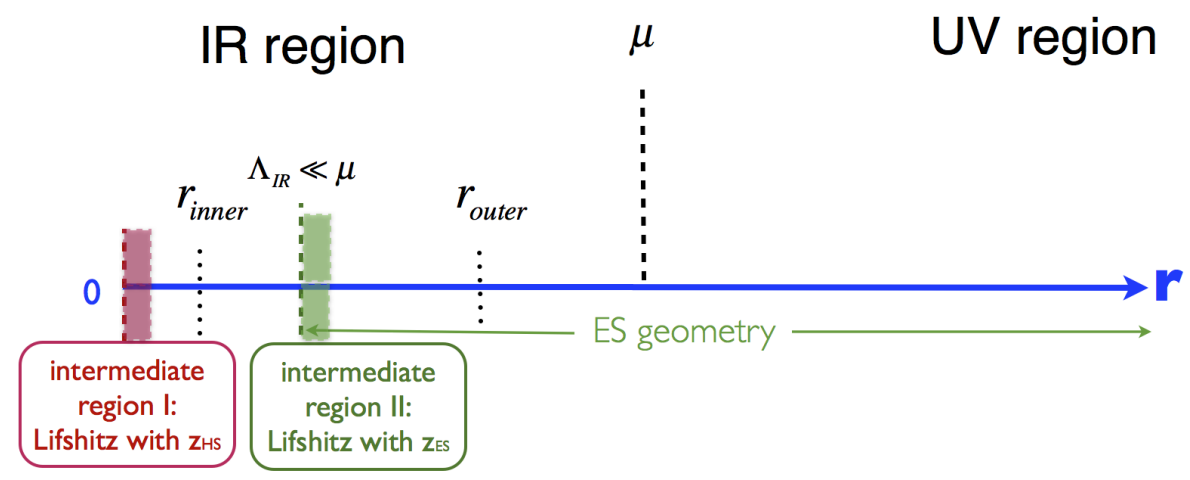

Figure 14. Transition scales for the two-edge hairy electron star. The near horizon area of the condensed phase is composed of two critical regions and relevant deformations in between. Intermediate region $\mathrm{I}$ is Lifshitz $z_{\mathrm{HS}}$ and intermediate region II is Lifshitz $z_{\mathrm{ES}}$.

for the one-edge HES. At this scale the geometry turns from the near horizon geometry of ES to the geometry of HES at this scale, which means that the inner edge of the two-edge HES solutions near the BF bound is also close to 0. At this inner edge, which can also be thought of as an emergent IR scale the solution becomes fully HS. This behavior is sketched in Fig. 14.

\subsection{The critical point}

The final noteworthy feature of the phase diagram is the quadruple critical point at which any two phases can be connected with each other. From this critical point, we can in principle realize quantum phase transitions directly between AdS-RN and HES or between HS and ES by traversing diagonally in the phase diagram through the critical point. This is quite interesting and we will leave this for future study. Here we wish to notice that the existence of the emergent IR scales resolves some of the paradoxes one might encounter when considering this situation. At the critical point, the AdS-RN solution is connected to the HS solution, so when we decrease $\hat{m}_{f}$ the phase transition from HS to HES should be the same as the phase transition from AdS-RN to ES. But because ES is always one-edge, this seems to be in contradiction with the fact that the phase transition from HS to HES at the critical point is to two-edge HES solution and one edge HES solutions only exist to the left of the red curve in Fig. 8. In fact, this "jump" is a direct consequence of the discrete difference in the near horizon geometry between RN and HS at the critical point. We saw before that this discreteness paradox gets resolved by the exponential suppression of the condensate away from the horizon. So it goes here. Although the ES solution at the critical point is one-edge, the HES solution at the critical point is still two-edge, but the inner edge is exponentially close to the horizon. 


\section{Conclusion and discussion}

The existence of a hairy AdS electron star solution as we have constructed in this paper shows that relativistic critical theories can support a mixed phase of bosons and fermions. Bosons don't always win in these set-ups, and we have co-existence rather than competition. The construction clearly shares a lot of characteristics with the conventional counterexample of relativistic heavy bosons with light fermions. In the bulk it is almost exactly what is taking place, whereas in the boundary conformal field theory the role of mass is played by the conformal dimension. One could have thought that the other simple counterexample, a very large number of fermion flavors such that one can circumvent the Pauli principle, is also at play as we know that the fluid approximation in the bulk essentially corresponds to a system with an infinite number of quasiparticles distinguished by their AdS radial quantum number. It is not clear that this is case. Based on our understanding of single Fermi surface holography [22-24, 26] most of the above story will apply in that situation as well. Moreover including $1 / N$ corrections in the bulk should render the higher order Fermi surfaces unstable while keeping the macroscopic characteristics of the star. Another criticism which one might raise is that it appears we are simply describing a non-interacting system of bosons and fermions. A reason to think so is that for incommensurate charges where $q_{b}$ and $q_{f}$ are not integer multiples of each other one can think of the system as having a separate $U(1)_{b}$ and $U(1)_{f}$ symmetry, where we are keeping the off-diagonal chemical potential to zero. ${ }^{11}$ We argue that this should also not apply on inspection. The direct argument is that the field theory described is interacting, albeit rearranged in a $1 / N$ perturbative series, and similarly the AdS side clearly experiences gravitational and electromagnetic interactions. Preliminary results in a forthcoming companion paper where we study the same system for $q_{b}=2 q_{f}$ with a Yukawa interaction fully support this view [19, 20].

\section{Acknowledgments}

We thank Hong Liu for very useful correspondence. We would also like to thank Steven Gubser and Richard Davison for helpful conversations. Y.L and Y.W. S would like to thank Rong-Gen Cai, Bin Chen, Yi Ling, Zheng-Yu Weng for discussions and they are very grateful to the hospitality of CHEP, Peking University and Shanghai Jiaotong University where this work was presented. This research is supported in part by a Spinoza Award (J. Zaanen) from the Netherlands Organization for Scientific Research (NWO) and by the Dutch Foundation for Fundamental Research on Matter (FOM).

\section{References}

[1] P. Basu, J. He, A. Mukherjee, M. Rozali and H. -H. Shieh, JHEP 1010, 092 (2010) [arXiv:1007.3480 [hep-th]].

[2] A. Donos, J. P. Gauntlett, J. Sonner and B. Withers, arXiv:1212.0871 [hep-th].

[3] D. Musso, JHEP 1306, 083 (2013) [arXiv:1302.7205 [hep-th]].

\footnotetext{
${ }^{11}$ We thank Steven Gubser for emphasizing this.
} 
[4] R. -G. Cai, L. Li, L. -F. Li and Y. -Q. Wang, arXiv:1307.2768 [hep-th].

[5] S. A. Hartnoll, C. P. Herzog and G. T. Horowitz, Phys. Rev. Lett. 101, 031601 (2008) [arXiv:0803.3295 [hep-th]].

[6] S. A. Hartnoll, C. P. Herzog and G. T. Horowitz, JHEP 0812, 015 (2008) [arXiv:0810.1563 [hep-th]].

[7] S. S. Gubser and A. Nellore, Phys. Rev. D 80, 105007 (2009) [arXiv:0908.1972 [hep-th]].

[8] G. T. Horowitz and M. M. Roberts, JHEP 0911, 015 (2009) [arXiv:0908.3677 [hep-th]].

[9] H. Liu, J. McGreevy and D. Vegh, Phys. Rev. D 83, 065029 (2011) [arXiv:0903.2477 [hep-th]].

[10] M. Cubrovic, J. Zaanen and K. Schalm, Science 325, 439 (2009) [arXiv:0904.1993 [hep-th]].

[11] T. Faulkner, H. Liu, J. McGreevy and D. Vegh, Phys. Rev. D 83, 125002 (2011) [arXiv:0907.2694 [hep-th]].

[12] T. Faulkner and J. Polchinski, JHEP 1106, 012 (2011) [arXiv:1001.5049 [hep-th]].

[13] S. A. Hartnoll and A. Tavanfar, Phys. Rev. D 83, 046003 (2011) [arXiv:1008.2828 [hep-th]].

[14] S. A. Hartnoll, D. M. Hofman and D. Vegh, JHEP 1108, 096 (2011) [arXiv:1105.3197 [hep-th]].

[15] N. Iqbal, H. Liu and M. Mezei, JHEP 1204, 086 (2012) [arXiv:1105.4621 [hep-th]].

[16] M. Cubrovic, Y. Liu, K. Schalm, Y. -W. Sun and J. Zaanen, Phys. Rev. D 84, 086002 (2011) [arXiv:1106.1798 [hep-th]].

[17] F. Nitti, G. Policastro and T. Vanel, arXiv:1307.4558 [hep-th].

[18] M. Edalati, K. W. Lo and P. W. Phillips, Phys. Rev. D 84, 066007 (2011) [arXiv:1106.3139 [hep-th]].

[19] Y. Liu, K. Schalm, Y. -W. Sun and J. Zaanen, work in progress.

[20] A. Bagrov, B. Meszena, K. Schalm, and J. Zaanen, work in progress.

[21] S. A. Hartnoll, J. Polchinski, E. Silverstein and D. Tong, JHEP 1004, 120 (2010) [arXiv:0912.1061 [hep-th]].

[22] S. Sachdev, Phys. Rev. D 84, 066009 (2011) [arXiv:1107.5321 [hep-th]].

[23] A. Allais, J. McGreevy and S. J. Suh, Phys. Rev. Lett. 108, 231602 (2012) [arXiv:1202.5308 [hep-th]].

[24] A. Allais and J. McGreevy, arXiv:1306.6075 [hep-th].

[25] M. V. Medvedyeva, E. Gubankova, M. ubrovi, K. Schalm and J. Zaanen, arXiv:1302.5149 [hep-th].

[26] M. Cubrovic, J. Zaanen and K. Schalm, JHEP 1110, 017 (2011) [arXiv:1012.5681 [hep-th]].

[27] N. Iqbal, H. Liu, M. Mezei and Q. Si, Phys. Rev. D 82, 045002 (2010) [arXiv:1003.0010 [hep-th]].

[28] J. de Boer, K. Papadodimas and E. Verlinde, JHEP 1010, 020 (2010) [arXiv:0907.2695 [hep-th]].

[29] X. Arsiwalla, J. de Boer, K. Papadodimas and E. Verlinde, JHEP 1101, 144 (2011) [arXiv:1010.5784 [hep-th]]. 
[30] V. G. M. Puletti, S. Nowling, L. Thorlacius and T. Zingg, JHEP 1101, 117 (2011) [arXiv:1011.6261 [hep-th]].

[31] S. A. Hartnoll and P. Petrov, Phys. Rev. Lett. 106, 121601 (2011) [arXiv:1011.6469 [hep-th]].

[32] S. A. Hartnoll and L. Huijse, arXiv:1111.2606 [hep-th].

[33] B. Gouteraux and E. Kiritsis, JHEP 1304, 053 (2013) [arXiv:1212.2625 [hep-th]].

[34] N. Iqbal, H. Liu and M. Mezei, arXiv:1108.0425 [hep-th].

[35] N. Iqbal and H. Liu, Class. Quant. Grav. 29, 194004 (2012) [arXiv:1112.3671 [hep-th]].

[36] L. Huijse and S. Sachdev, Phys. Rev. D 84, 026001 (2011) [arXiv:1104.5022 [hep-th]].

[37] T. Faulkner, G. T. Horowitz and M. M. Roberts, JHEP 1104, 051 (2011) [arXiv:1008.1581 [hep-th]].

[38] S. Franco, A. Garcia-Garcia and D. Rodriguez-Gomez, JHEP 1004, 092 (2010) [arXiv:0906.1214 [hep-th]]. 